\title{
Bioactive Compounds Produced by Strains of Penicillium and Talaromyces of Marine Origin
}

\author{
Rosario Nicoletti ${ }^{1, *,+}$ and Antonio Trincone ${ }^{2}$ \\ 1 Council for Agricultural Research and Agricultural Economy Analysis, Rome 00184, Italy \\ 2 Institute of Biomolecular Chemistry, National Research Council, Pozzuoli 80078, Italy; \\ antonio.trincone@icb.cnr.it \\ * Correspondence: rosario.nicoletti@entecra.it; Tel.: +39-081-253-9194 \\ † Current address: Department of Agriculture, University of Naples "Federico II", Portici 80055, Italy. \\ Academic Editor: Orazio Taglialatela-Scafati \\ Received: 23 December 2015; Accepted: 25 January 2016; Published: 18 February 2016
}

\begin{abstract}
In recent years, the search for novel natural compounds with bioactive properties has received a remarkable boost in view of their possible pharmaceutical exploitation. In this respect the sea is entitled to hold a prominent place, considering the potential of the manifold animals and plants interacting in this ecological context, which becomes even greater when their associated microbes are considered for bioprospecting. This is the case particularly of fungi, which have only recently started to be considered for their fundamental contribution to the biosynthetic potential of other more valued marine organisms. Also in this regard, strains of species which were previously considered typical terrestrial fungi, such as Penicillium and Talaromyces, disclose foreground relevance. This paper offers an overview of data published over the past 25 years concerning the production and biological activities of secondary metabolites of marine strains belonging to these genera, and their relevance as prospective drugs.
\end{abstract}

Keywords: bioactive metabolites; chemodiversity; marine fungi; Penicillium; Talaromyces

\section{Introduction}

For a long time fungi have been considered as a fundamentally terrestrial form of life. In the past few decades, this concept has started to be revised based on the emerging evidence that these microorganisms are also widespread in the marine habitat. New species recovered from marine substrates are reported repeatedly, which makes a reliable estimate of their actual number quite problematic [1]. Attention of researchers in the field often tends to be focused on the obligate marine species, defined for their ability to grow and sporulate exclusively in a marine habitat [2]. However, it is a matter of fact that many species found at sea are already known from terrestrial contexts, which makes their placement in a "facultative" category more appropriate. It is obvious that the mere isolation from a marine substrate does not imply a real adaptation of a fungus to develop in those particular conditions. Nevertheless, this aspect becomes secondary when considering discovery and exploitation of bioactive compounds, and in view of this objective the ecological versatility of the facultative marine fungi introduces them as being among the most valuable natural resources, deserving to be better characterized through more detailed genetic and biochemical analyses [3].

The issue of bioactive compound production is fundamental in understanding the complex ecological relationships established among and between sea-inhabiting organisms and microorganisms, and presents human nutritional implications due to the possibility that such fungal strains contaminate sea food, and their metabolites eventually act as mycotoxins [4,5]. However, the pharmaceutical industry can be regarded as the application field where products from marine fungi have the most substantial impact, since many of them have entered the clinical pipeline in view of being exploited 
as novel drugs. Quantitative considerations about fruitfulness in the discovery of new metabolites show that the number of compounds obtained from marine-derived fungi is increasing at a high rate. From a total of about 270 known before 2002, investigations in the field have added more than 800 such products up to 2010 [6], as a result of the availability of bioassay-guided fractionation systems, the accessibility to higher field NMR and mass spectrometers, and the development of the so-called hyphenated spectroscopy technologies (HPLC-MS, HPLC-NMR, etc.) [7]. The recent combining of natural product chemistry and metabolomic approaches in drug discovery can certainly contribute to the development of new leads from marine derived fungi [8].

Within the facultative marine fungi, species of Penicillium and Talaromyces are particularly known for their ability to produce important bioactive compounds. This paper offers an overview of the literature issued in the past 25 years concerning production and biological activities of secondary metabolites of marine strains belonging to the above genera, and their relevance as prospective drugs. Our review basically considers strains obtained from marine sources in a topographic sense, thus possibly including strains/species whose occurrence at sea is merely incidental. Conversely, we did not treat isolates from mangrove plants and their rhizosphere, whose connection with the sea is more remote, and probably deserve a dedicated review. As for the compounds, this overview does not consider primary metabolites mentioned in the cited references, including ergosterol and structurally related compounds $[9,10]$. Additional exclusions concern other common compounds which often represent intermediates in the synthesis of more complex secondary metabolites, such as orsellinic acid [11,12], and products obtained from mutant strains [13-15], or through co-cultivation of two or more strains [16].

\section{Penicillium and Talaromyces: An Extraordinary Source of Bioactive Compounds}

The Ascomycetous genus Talaromyces (Eurotiomycetes, Trichocomaceae) was initially designated to comprise the teleomorphs of a number of biverticillate Penicillium species. However, following the principle "one fungus-one name" recently affirmed in fungal taxonomy, by which a single holomorphic denomination is to be adopted for species presenting two alternating stages in their life cycle [17], the concept of Talaromyces has been recently extended to include all species in the Penicillium subgenus Biverticillium [18], while the name Penicillium is conserved sensu stricto for species belonging to the subgenera Aspergilloides, Furcatum, and Penicillium, for their associated Eupenicillium teleomorphs, and for species previously classified in a few related genera [19]. Information concerning production of secondary metabolites also supports the separation of the two genera in distinct monophyletic groups based on DNA sequencing [20], and the accumulation of novel data provides a remarkable contribution under the taxonomic viewpoint, particularly in view of a correct species ascription of the many strains which are provisionally reported as Penicillium/Talaromyces sp. [18,21].

Traditionally, species in the genus Penicillium and Talaromyces, which are fundamentally saprophytic and ubiquitary, have been regarded as a fruitful investigational ground for the finding of novel bioactive compounds, leading to the discovery of blockbuster drugs, such as penicillin [22] and the anticholesterolemic agent compactin [23], miscellaneous antitumor products [24], and mycotoxins contaminating food [25]. Most of these fundamental studies were carried out on strains from soil and food commodities. Thus, in a way it is not surprising that the number of bioactive compounds is continuously increasing when a so far inadequately explored context such as the sea has become the subject of systematic investigations. This inference is particularly valid for the species treated in this paper, considering that in a recent review Penicillium is reported as the second most common genus of marine fungi [26]. In our overview we recorded over 550 compounds, or compound families, from a total of about 150 strains belonging to 39 species of Penicillium and five species of Talaromyces (Table 1). Unclassified strains, referred to as Penicillium sp. or Talaromyces sp., represent a remarkable share (ca. 38\%), which implies that the number of marine species within these genera is destined to increase when and if more work is carried out on some of these strains, eventually leading to their correct species ascription. With reference to this taxonomic aspect, the characterization of two novel species, Penicillium marinum [25] and Penicillium dravuni [27], deserves to be particularly mentioned. 
Table 1. Secondary metabolites of Penicillium and Talaromyces strains of marine origin. List is based on the chronological order of isolation of the producing strains.

\begin{tabular}{|c|c|c|c|c|c|}
\hline Species Name $^{1}$ & Strain No. & Source of Isolation ${ }^{2}$ & Location & Products ${ }^{3}$ & References \\
\hline $\begin{array}{l}\text { P. fellutanum } \\
\text { (P. dierckxii) }\end{array}$ & - & F Apogon endekataenia & Manazaru (Japan) & $\underline{\text { Fellutamide A-B }}$ & [28] \\
\hline $\begin{array}{l}\text { Penicillium sp. } \\
\text { (P. marinum })\end{array}$ & OUPS-79 & $\begin{array}{l}\text { G Ulva (Enteromorpha) } \\
\text { intestinalis }\end{array}$ & Tanabe Bay (Japan) & $\begin{array}{l}\text { Communesin A-B, Penochalasin A-H, Penostatin A-I, } \\
\text { Chaetoglobosin A,F,O, Patulin, Epiepoxydon }\end{array}$ & [29-34] \\
\hline P. citrinum & - & $\mathrm{S}$ unidentified sponge & Suruga Bay (Japan) & Cathestatin A-B, Estatin A-B & [35] \\
\hline Penicillium sp. & BM1689-P & sediment & Uchiura Bay (Japan) & Epolactaene & [36] \\
\hline Penicillium sp. & BM923 & sediment & Miho (Japan) & Acetophthalidin, 3,4,6-Trihydroxymellein & [37] \\
\hline Penicillium sp. & - & intertidal sediment & $\begin{array}{l}\text { San Antonio Oeste } \\
\text { (Argentina) }\end{array}$ & Cyclo(L-prolyl-L-tyrosyl) & [38] \\
\hline Penicillium sp. & NI15501 & sediment (depth $14 \mathrm{~m}$ ) & Tomari (Japan) & $\underline{\text { NI15501A }}$ & [39] \\
\hline P. waksmanii & OUPS-N133 & B Sargassum ringgoldianum & Japan & Pyrenocine A-B,D-E, Cis-bis(methylthio)silvatin & [40] \\
\hline P. citrinum & many strains & several sources & $\begin{array}{l}\text { Mochima Bay and Paria } \\
\text { Bay (Venezuela) }\end{array}$ & Citrinin, Tanzawaic acid A & {$[41,42]$} \\
\hline P. steckii & $\begin{array}{l}\text { M23B-7 = IBT20952 and } \\
12 \text { more strains }\end{array}$ & $\begin{array}{l}\text { T unidentified tunicate, } \\
\text { and other sources } \\
\text { (molluscs, fish, sponges) }\end{array}$ & $\begin{array}{l}\text { Mochima Bay and Paria } \\
\text { Bay (Venezuela) }\end{array}$ & $\begin{array}{l}\text { Tanzawaic acid E-F, 3,7-Dimethyl-8-hydroxy-6-methoxyisochroman, } \\
\text { 3,7-Dimethyl-1,8-dihydroxy-6-methoxyisochroman }\end{array}$ & {$[41,42]$} \\
\hline Penicillium sp. & \#CNC-350 & G Avrainvillea longicaulis & Sweetings Cay (Bahamas) & $\begin{array}{l}\text { Verticillin A, 11'-Deoxyverticillin A, } 11,11^{\prime} \text {-Dideoxyverticillin A, } \\
\text { Bisdethio-bis(methylthio)-dioxopiperazine }\end{array}$ & [43] \\
\hline Penicillium sp. & K029 & M Mytilus coruscus & Seragaki (Japan) & Coruscol A, Herquline A & [44] \\
\hline Penicillium sp. & K036 & M M. coruscus & Seragaki (Japan) & $\underline{\text { Sculezonone A-B }}$ & [45] \\
\hline Penicillium sp. & $\# 386$ & sand & South China Sea & Penicillazine (Trichodermamide A) & [46] \\
\hline P. cf. montanense & $\mathrm{HBI}-3 / \mathrm{D}$ & S Xestospongia exigua & $\begin{array}{l}\text { Mangangan Island } \\
\text { (Indonesia) }\end{array}$ & $\underline{\text { Xestodecalactone } \mathrm{A}-\mathrm{C}}$ & [47] \\
\hline P. citrinum & 991084 & $\mathbf{S}$ Axinella sp. & Papua New Guinea & Isocyclocitrinol A, 22-Acetylisocyclocitrinol A & [48] \\
\hline P. brocae & F97S76 & S Zyzzya sp. & Fiji & $\underline{B r o c a e n o l ~ A-C}$ & [49] \\
\hline $\begin{array}{l}\text { Penicillium sp. } \\
\text { (P. dravuni) }\end{array}$ & F01V25 & G Dictyosphaeria versluyii & Dravuni (Fiji) & Dictyosphaeric acid A-B, Carviolin & [50] \\
\hline $\begin{array}{l}\text { Penicillium sp. } \\
\text { (P. marinum) }\end{array}$ & E-00-12/3 & $\mathbf{S}$ Axinella verrucosa & Elba Island (Italy) & Communesin B,C-D, Griseofulvin, Dechlorogriseofulvin, Oxaline & [51] \\
\hline $\begin{array}{c}\text { P. cf. } \\
\text { brevicompactum }\end{array}$ & E-00-2/6a & $\mathbf{S}$ Petrosia ficiformis & Elba Island (Italy) & Petrosifungin A-B, Brevianamide A, Asperphenamate, Mycophenolic acid & [52] \\
\hline Penicillium sp. & a004181, b004181 & sediment (depth $4380 \mathrm{ft}$ ) & Matuka (Fiji) & $\begin{array}{l}\text { Anserinone A-B, Formylanserinone B, Epoxyserinone A-B, Deoxyanserinone B, } \\
\text { Hydroxymethylanserinone B }\end{array}$ & [53] \\
\hline
\end{tabular}


Table 1. Cont.

\begin{tabular}{|c|c|c|c|c|c|}
\hline Species Name $^{1}$ & Strain No. & Source of Isolation $^{2}$ & Location & Products $^{3}$ & References \\
\hline $\begin{array}{l}\text { P. waksmanii } \\
\text { (Penicillium sp.) }\end{array}$ & LCP99.43.43 = MMS351 & water & La Prée (France) & $\begin{array}{l}\text { Griseofulvin, Dechlorogriseofulvin, Orcinol, Penicillic acid, Agroclavine, } \\
\text { Festuclavine, Nortryptoquivaline, Ligerin }\end{array}$ & [54-56] \\
\hline P. citrinum & N059 & $\mathbf{R}$ Actinotrichia fragilis & Okinawa (Japan) & Citrinin, Citrinadin A-B & {$[57,58]$} \\
\hline P. citrinum & N055 & F Scarus ovifrons & Okinawa (Japan) & Perinadine A, Scalusamide A-C & {$[59,60]$} \\
\hline P. janczewskii & H-TW5/869 & water & $\begin{array}{l}\text { Helgoland Island } \\
\text { (Germany) }\end{array}$ & $\begin{array}{l}\text { 3,4-Dihydroxy-4-(4'-methoxyphenyl)-3,4-dihydro-2(1H)-quinolinone, } \\
\text { Peniprequinolone, } \\
\text { 3-Methoxy-4-hydroxy-4-(4'-methoxyphenyl)-3,4-dihydro-2(1H)-quinolinone }\end{array}$ & [61] \\
\hline P. chrysogenum & DSM16137 = E01-10/3 & S Ircinia fasciculata & Elba Island (Italy) & $\begin{array}{l}\text { Sorbicillactone A-B, Sorbivinetone, } \\
\text { Bisvertinolone, Sorbicillin, Oxosorbicillinol, Meleagrin, Roquefortine C-D }\end{array}$ & {$[62,63]$} \\
\hline $\begin{array}{c}\text { P. terrestre } \\
\text { (P. crustosum) }\end{array}$ & M204077 & sediment & Jiaozhou Bay (China) & $\begin{array}{l}\text { Sorbicillin, Dihydrobisvertinolone, Tetrahydrobisvertinolone, Trichodimerol, } \\
\text { Dihydrotrichodimerol, Tetrahydrotrichodimerol, Sorbiterrin A, Penicillone A-B, } \\
\text { Chloctanspirone A-B, Terrestrol A-H,K-L, } \\
\text { 2-(2'-3'-Dihydrosorbyl)-3,6-dimethyl-5-hydroxy-1,4-benzoquinone, } \\
\text { 3-Acetonyl-2,6-dimethyl-5-hydroxy-1,4-benzoquinone }\end{array}$ & [64-69] \\
\hline P. janthinellum & - & C Dendronephyta sp. & Hainan (China) & Griseofulvin, Dechlorogriseofulvin, Janthinolide A-B, Deoxymycelianamide & [70] \\
\hline P. brevicompactum & $\mathrm{Cl}-2002$ & S Cliona sp. & Quintay (Chile) & Mycophenolic acid, Mycophenolic acid methyl ester, Tyrosol & [71] \\
\hline $\begin{array}{l}\text { P. rugulosum } \\
\text { (T. rugulosus) }\end{array}$ & KF021 & S Chondrosia reniformis & Elba Island (Italy) & Prugosene A1,A2,B1,B2,B3,C1,C2 & [72] \\
\hline Penicillium sp. & - & B Sargassum tortile & Toyama Bay (Japan) & $\begin{array}{l}\text { 4-Hydroxy-2-methoxyacetanilide, 4-Methoxyphenylacetic acid, } \\
\text { 4-(2-Hydroxyethyl)phenol, 3-Methoxyphenol, 4-Hydroxyphenylacetic acid }\end{array}$ & [73] \\
\hline P. janthinellum & - & sediment (depth $11 \mathrm{~m}$ ) & $\begin{array}{l}\text { Amursky Bay } \\
\text { (Sea of Japan) }\end{array}$ & Shearinine $\mathrm{A}, \mathrm{D}-\mathrm{F}$ & [74] \\
\hline P. bilaiae & MST-MF667 & boat ramp & $\begin{array}{l}\text { Port Huon } \\
\text { (Tasmania, Australia) }\end{array}$ & $\begin{array}{l}\text { Cyclo(L-prolyl-L-tyrosyl), Cyclo(L-phenalanyl-L-prolyl), Cyclo(L-prolyl-L-valyl), } \\
\text { Cis-bis(methylthio)silvatin, Bilain A-C, Pistillarin, Citromycin, } \\
\text { 2,3-Dihydrocitromycin, Citromycetin, 2,3-Dihydrocitromycetin }\end{array}$ & [75] \\
\hline Penicillium sp. & MFA446 & G Ulva pertusa & Bijin Island (Korea) & Citrinin, Citrinin H2, Redoxcitrinin, Phenol A, Phenol A acid, 4-Hydroxymellein & [76] \\
\hline P. aurantiogriseum & SP0-19 & S Mycale plumose & Jiaozhou Bay (China) & Aurantiomide A-C, Anacin & [77] \\
\hline $\begin{array}{c}\text { P. stoloniferum } \\
\text { (P. brevicompactum) }\end{array}$ & QY2-10 & $\mathbf{T}$ unidentified ascidian & Jiaozhou Bay (China) & $\underline{\text { Stoloniferol A-B }}$ & [78] \\
\hline $\begin{array}{l}\text { P. flavidorsum } \\
\text { (P. glabrum) }\end{array}$ & SHK1-27 & sediment & Weizhou Island (China) & $\begin{array}{l}\text { Averufin, 8-O-Methylaverufin, 6,8-O-Dimethylaverufin, Averantin, Nidurufin, } \\
\text { Versicolorin A-B, Versiconol }\end{array}$ & [79] \\
\hline $\begin{array}{l}\text { P. minioluteum } \\
\text { (T. minioluteus) }\end{array}$ & 03HE3-1 & mud & Heita Bay (Japan) & Miniolutelide A-B, 22-Epoxyberkeleydione & [80] \\
\hline Penicillium sp. & BL27-2 & mud & Bering Sea & $\begin{array}{l}\text { 3-Acetyl-13-deoxyphomenone, } 8 \alpha \text {-Hydroxy-13-deoxyphomenone, } \\
\text { Sporogen-AO1, 3-Acetyl-9,7(11)-dien-7 } \alpha \text {-hydroxy-8-oxoeremophilane, } \\
\text { 6-Dehydropetasol, 7-Hydroxypetasol }\end{array}$ & [81] \\
\hline Penicillium sp. & SS080624SCf1 & T Didemnum molle & Ishigaki Island (Japan) & Phomenone, Sporogen-AO1, JBIR-27, JBIR-28 & [82] \\
\hline $\begin{array}{l}\text { Penicillium sp. } \\
\text { (Talaromyces sp.) }\end{array}$ & AF1-2 & salt pan & Australia & 3-O-Methylfunicone & [83] \\
\hline
\end{tabular}


Table 1. Cont

\begin{tabular}{|c|c|c|c|c|c|}
\hline Species Name $^{1}$ & Strain No. & Source of Isolation ${ }^{2}$ & Location & Products ${ }^{3}$ & References \\
\hline Penicillium sp. & CANU MCPT14-1-5 & B Xiphophora gladiata & Otago (New Zealand) & PF1140, Deoxy-PF1140, Deoxyakanthomycin & {$[84]$} \\
\hline Penicillium sp. & i-1-1 & G Blidingia minima & Yantai (China) & Citrinin, Citrinal A, 2,3,4-Trimethyl-5,7-dihydroxy-2,3-dihydrobenzofuran & [85] \\
\hline Penicillium sp. & EG-51 & G Ulva sp. & Suez Canal (Egypt) & Chromanone A & [86] \\
\hline Penicillium sp. & F1 & sediment (depth $5080 \mathrm{~m})$ & Pacific Ocean & Brevicompanine B, $\underline{\mathrm{D}-\mathrm{H}}$, Fructigenine $\mathrm{B}$ & [87] \\
\hline Penicillium sp. & F23-2 & sediment (depth $5080 \mathrm{~m})$ & Pacific Ocean & $\begin{array}{l}\text { Meleagrin B-E, Roquefortines F-I, Conidiogenone B-G, Sorbicillamine A-E, } \\
\text { Bisvertinolone, Rezishanone C, Penicyclone A-E }\end{array}$ & [88-91] \\
\hline Penicillium sp. & 3A00005 & sediment (depth $5115 \mathrm{~m}$ ) & East Pacific Ocean & Brevione A-B,F-K, Sterolic acid & {$[92,93]$} \\
\hline P. expansum & MMS42 & sediment & Le Croisic (France) & $\begin{array}{l}\text { Communesin A-B, D-E, Com } 470, \text { Com512, Com522, Com524, Com570, Com622, } \\
\text { Com 644, Patulin, Chaetoglobosin 528, Chaetoglobosin 530, Citrinin, } \\
\text { Roquefortine C-D, Expansolide A-B, Aurantioclavine, Verruculotoxin }\end{array}$ & {$[54,94]$} \\
\hline Penicillium sp. & PSU-F44 & C Annella sp. & Similan Islands (Thailand) & Penicipyrone, Penicilactone, Brefeldin A,C, Oxobrefeldin A & [95] \\
\hline Penicillium sp. & PSU-F40 & C Annella sp. & Similan Islands (Thailand) & $\begin{array}{l}\text { Penicipyrone, Penicipyranone, Penicisochroman A-E, Penicisoquinoline, } \\
\text { Peniciphenol, TMC-120B, TMC-120C, 2-(2-Methoxybenzoyl)pyrrole, } \\
\text { 1-(2,4-Dihydroxy-6-methylphenyl)-3-methyl-1-butanone, Nicotinic acid }\end{array}$ & [96] \\
\hline Penicillium sp. & M207142 & sediment & China & $\begin{array}{l}(2 E, 4 E)-1-(2,6-\text { Dihydroxy-3,5-dimethyl-phenyl)hexa-2,4-dien-1-one, } \\
\mathrm{A}, 2^{\prime}, 3^{\prime} \text {-Dihydrosorbicillin }\end{array}$ & [97] \\
\hline P. chrysogenum & R03-8/4 = LF066 & $\mathbf{S}$ Tethya aurantium & Limsky Canal (Croatia) & $\begin{array}{l}\text { Meleagrin, Roquefortine C-D, Sorbifuranone B-C, Bisvertinolone, } \\
2^{\prime}, 3^{\prime} \text {-Dihydrosorbicillin, Xanthocillins, Cillifuranone }\end{array}$ & {$[63,98]$} \\
\hline P. citrinum & SpI080624G1f01 & $\begin{array}{l}\text { S unidentified } \\
\text { Demospongia }\end{array}$ & Ishigaki Island (Japan) & $\begin{array}{l}\text { Redoxcitrinin, Sclerotinin A-B, Bisorbibutenolide, Bisvertinolone, Trichodimerol, } \\
\text { JBIR-59, JBIR-124 }\end{array}$ & {$[99,100]$} \\
\hline P. oxalicum & $\mathrm{F} 30=\mathrm{CBMAI} 1185$ & G Caulerpa sp. & Sao Paulo State (Brazil) & Meleagrin, Oxaline & {$[101]$} \\
\hline P. citrinum & F53 = CBMAI1186 & G Caulerpa sp. & Sao Paulo State (Brazil) & $\begin{array}{l}\text { Citrinin, Citrinalin A-B, } \\
\text { (E)-1-(2,3-dihydro-1H-pyrrol-1-yl)-2-methyldec-8-ene-1,3-dione, } \\
\text { 1-(2,3-dihydro-1H-pyrrol-1-yl)-2-methyldecane-1,3-dione }\end{array}$ & [101] \\
\hline P. griseofulvum & - & sediment (depth $2481 \mathrm{~m})$ & Pacific Ocean & $\begin{array}{l}\text { Echinulin, Preechinulin, Didehydroechinulin, Isoechinulin B, Neoechinulins A-B, } \\
\text { Tardioxopiperazine A, Variecolorin H, } \underline{\mathrm{M}-\mathrm{O}}\end{array}$ & [102] \\
\hline P. aurantiogriseum & MF361 & mud & Bohai Sea (China) & $\begin{array}{l}\text { Verrucosidin, Norverrrucosidin, Verrucosidinol, Verrucosidinol acetate, Terrestric } \\
\text { acid, Aurantiomide C, Auranthine, Auranomide A-C }\end{array}$ & {$[103,104]$} \\
\hline P. chrysogenum & MFB574-2 & $\mathbf{R}$ Hypnea species complex & Yokgee Island (Korea) & 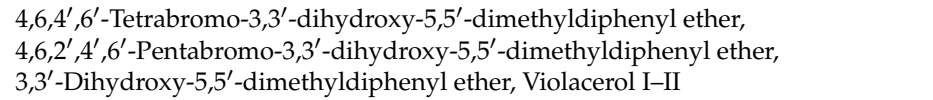 & [105] \\
\hline Penicillium sp. & CNL-338 & $\mathbf{R}$ Laurencia sp. & Bahamas & Penilumamide, Aspochalasin D-E & [106] \\
\hline P. chrysogenum & QEN-24S & $\mathbf{R}$ Laurencia sp. & Weizhou Island (China) & $\begin{array}{l}\text { Penicitide A-B, Penicimonoterpene, Penicisteroid A-B, Conidiogenol, } \\
\text { 2-(2,4-Dihydroxy-6-methylbenzoyl)-glycerol, Anicequol, } \\
\text { 1-(2,4-Dihydroxy-6-methylbenzoyl)-glycerol, Conidiogenone B-D,F, } \underline{\mathrm{H}-\mathrm{I}}\end{array}$ & [107-109] \\
\hline P. glabrum & - & P Zostera marina (stem) & Trinity Bay (Sea of Japan) & Sulochrin, 4-Methoxy-3-methylgoniothalamin & [110] \\
\hline P. implicatum & - & P Z. marina (rhizome) & Trinity Bay (Sea of Japan) & Sulochrin, 4-Methoxy-3-methylgoniothalamin & [110] \\
\hline
\end{tabular}


Table 1. Cont

\begin{tabular}{|c|c|c|c|c|c|}
\hline Species Name $^{1}$ & Strain No. & Source of Isolation $^{2}$ & Location & Products $^{3}$ & References \\
\hline P. citrinum & - & sediment & Langqi Island (China) & $\begin{array}{l}\text { Citrinin, Decarboxydihydrocitrinone, Penicitrinol C-E, } \\
\text { Dicitrinone B, Penicitrinine A }\end{array}$ & [111-113] \\
\hline Penicillium sp. & JMF034 & sediment (depth $1151 \mathrm{~m}$ ) & Suruga Bay (Japan) & $\begin{array}{l}\text { Gliotoxin, Gliotoxin G, 5a,6-Didehydrogliotoxin, } \\
\text { 6-Deoxy-5a,6-didehydrogliotoxin, } \\
\text { Bis(dethio)-10a-methylthio-3a-deoxy-3,3a-didehydrogliotoxin, } \\
\text { Bis(dethio)bis(methylthio)gliotoxin, } \\
\text { Bis(dethio)bis-(methylthio)-5a,6-didehydrogliotoxin }\end{array}$ & [114] \\
\hline P. brevicompactum & LF259 & S T. aurantium & Limsky Canal (Croatia) & Mycophenolic acid & [98] \\
\hline P. citreoviride & LF590 & S T. aurantium & Limsky Canal (Croatia) & Citreoviridins, Territrem B & [98] \\
\hline $\begin{array}{l}\text { P. canescens } \\
\text { (Penicillium sp.) }\end{array}$ & LF596 & $\mathbf{S}$ T. aurantium & Limsky Canal (Croatia) & Griseofulvin, Fiscalin A-C, Tryptoquivalin, Nortryptoquivalin & [98] \\
\hline P. sclerotiorum & LF607 & S T. aurantium & Limsky Canal (Croatia) & Sclerotiorin, Sclerotioramin, Azaphilone derivative (comp. D) & [98] \\
\hline Penicillium sp. & J05B-3-F-1 & S Stelletta sp. & Jeju Island (Korea) & $\begin{array}{l}\text { (3S)-Hexylitaconic acid, } \\
\text { (3S,8R)-Methyl 8-hydroxy-3-methoxycarbonyl-2-methylenenonanoate, } \\
\text { (3S,8R)-8-Hydroxy-3-carboxy-2-methylenenonanoic acid, } \\
\text { (3S)-9-Hydroxy-3-carboxy-2-methylenenonanoic acid, } \\
\text { (3S)-Methyl-9-hydroxy-3-methoxycarbonyl-2-methylenenonanoate }\end{array}$ & [115] \\
\hline P. paneum & SD-44 & sediment (depth $20 \mathrm{~m}$ ) & South China Sea & $\begin{array}{l}\text { Penipanoid A-C, 2-(4-Hydroxybenzyl)quinazolin-4(3H)-one, } \\
\text { Penipacid A-E, Penipaline A-C, } \\
\text { (-)-(3S)-2,3,4,9-Tetrahydro-1,1-dimethyl-1H- } \beta \text {-carboline-3-carboxylic acid, } \\
\text { 1,7-Dihydro-7,7-dimethylpyrano[2,3-g]indole-3-carbaldehyde }\end{array}$ & [116-118] \\
\hline P. commune & QSD-17 & sediment & South China Sea & $\begin{array}{l}\text { Meleagrin, Asperamide B1, Citreohybridonol, 3-Deacetylcitreohybridonol, } \\
\text { Comazaphilone A-F, Isophomenone, Conidiogenone B-D,F, Conidiogenol }\end{array}$ & {$[12,119]$} \\
\hline Penicillium sp. & $\mathrm{DG}(\mathrm{M} 3) 6^{\prime} \mathrm{C}$ & C Didemnum granulatum & Toque Island (Brazil) & 13-Desoxyphomenone & {$[120]$} \\
\hline P. raistrickii & $\mathrm{AC}(\mathrm{M} 2) 14$ & S Axinella cf. corrugata & Toque Island (Brazil) & Norlichexanthone & [120] \\
\hline P. paxilli & $\mathrm{Ma}(\mathrm{G}) \mathrm{K}$ & S Mycale angulosa & Toque Island (Brazil) & Pyrenocine $\mathrm{A}-\mathrm{B}, \mathrm{J}$ & [120] \\
\hline P. steckii & $\mathrm{AS}(\mathrm{F}) 39$ & B Sargassum sp. & Toque Island (Brazil) & 8-Methoxy-3,5-dimethylisochroman-6-ol & [120] \\
\hline Penicillium sp. & ghq208 & sediment & Jiaozhou Bay (China) & Penicinoline, Penicinoline E, Methylpenicinoline, Quinolactacide & [121] \\
\hline $\begin{array}{l}\text { P. pinophilum } \\
\text { (T. pinophilus) }\end{array}$ & - & G Ulva fasciata & Kasai Marine Park (Japan) & Pinophilin A-B, Sch725680 & [122] \\
\hline Penicillium sp. & $\mathrm{fS} 36$ & S unidentified sponge & Takarajima Island (Japan) & $\underline{\text { JBR-113, }}, \underline{-114}, \underline{-115}$ & [123] \\
\hline Penicillium sp. & F00120 & sediment (depth $1300 \mathrm{~m}$ ) & South China Sea & $\underline{\text { Penicilliumin A }}$ & [9] \\
\hline Penicillium sp. & SOF07 & sediment (depth $675 \mathrm{~m})$ & South China Sea & Mycophenolic acid, Hydroxy-mycophenolic acid, Penicacid A-C & [124] \\
\hline P. crustosum & PRB-2 & sediment (depth $526 \mathrm{~m})$ & Prydz Bay (Antarctica) & Penilactone A-B $, 2^{\prime}, 4^{\prime}$-Dihydroxy-3'-methoxymethyl-5'-methylacetophenone & [125] \\
\hline P. commune & SD-118 & sediment & South China Sea & $\begin{array}{l}\text { Meleagrin, Chrysogine, Methyl 2-N-(2-hydroxyphenyl)carbamoylacetate, } \\
\text { Asperamide A-B, Xanthocillin X, } N \text {-(2-Hydroxypropanoyl)-2-amino benzoic acid } \\
\text { amide, } N \text {-(2-Hydroxyphenyl)acetamide, } 4 \text {-Hydroxy benzaldehyde, } \\
\text { Methyl-2-(2-(1H-indol-3-yl)ethyl)carbamoyl)acetate, } N 2^{\prime} \text {-Acetyltryptophan } \\
\text { methyl ester, } N \text {-Acetyldopamine }\end{array}$ & [126] \\
\hline
\end{tabular}


Table 1. Cont

\begin{tabular}{|c|c|c|c|c|c|}
\hline Species Name ${ }^{1}$ & Strain No. & Source of Isolation $^{2}$ & Location & Products ${ }^{3}$ & References \\
\hline P. commune & 518 & C Muricella abnormalis & Danzhou (Hainan, China) & $\begin{array}{l}\text { Communol A-G, Clavatol, 2,4-Dihydroxy-3-methylacetophenone, } \\
\text { 2,4-Dihydroxy-3-methoxymethyl-5-methylacetophenone, } \\
\text { 2,4-Dihydroxy-5-methylacetophenone, cis-Bis(methylthio)silvatin }\end{array}$ & [127] \\
\hline P. canescens & MMS194 & water & La Baule (France) & $\begin{array}{l}\text { Griseofulvin, Dechlorogriseofulvin, Oxaline, Maculosin, Penicillic acid, } \\
\text { Penitremone A-C }\end{array}$ & [54] \\
\hline P. canescens & MMS460 & sediment & Le Croisic (France) & $\begin{array}{l}\text { Griseofulvin, Dechlorogriseofulvin, Oxaline, Penicillic acid, } \\
\text { Penitremone A-C }\end{array}$ & [54] \\
\hline Penicillium sp. & MMS747 & sediment & La Couplasse (France) & $\begin{array}{l}\text { Griseofulvin, Dechlorogriseofulvin, Penicillic acid, Nortryptoquivaline, } \\
\text { Agroclavine, Festuclavine }\end{array}$ & [54] \\
\hline P. chrysogenum & MMS5 & M shellfish & Le Croisic (France) & $\begin{array}{l}\text { Meleagrin, Roquefortine C-D, Chrysogine, Aurantioclavine, Maculosin, } \\
\text { Glandicolin A-B, Terrestric acid, Verruculotoxin }\end{array}$ & [54] \\
\hline P. antarcticum & MMS14 & $\mathbf{M}$ cockles & Le Croisic (France) & $\begin{array}{l}\text { Chrysogine, Cladosporin(=asperentin), 5-Hydroxyasperentin, Antarone A, } \\
\text { Violaceic acid, Patulin, Terrestric acid }\end{array}$ & {$[54,128]$} \\
\hline P. antarcticum & MMS15 & $\mathbf{M}$ cockles & Le Croisic (France) & $\begin{array}{l}\text { Chrysogine, Cladosporin, 5-Hydroxyasperentin, Aurantioclavine, Antarone A, } \\
\text { Patulin, Terrestric acid }\end{array}$ & {$[54,128]$} \\
\hline P. antarcticum & MMS163 & M mussel & Loire estuary (France) & Patulin, Chrysogine, Cladosporin, 5-Hydroxyasperentin, Terrestric acid & [128] \\
\hline P. marinum & MMS266 & M mussel & La Baule (France) & Penostatin derivatives, Fusoxysporone & [128] \\
\hline P. restrictum & MMS417 & $\mathbf{M}$ cockles & Le Croisic (France) & $\begin{array}{l}\text { Pestalotin, Hydroxypestalotin, } \\
\text { 5,6-Dihydro-4-methoxy-6-(1-oxopentyl)-2H-pyran-2-one }\end{array}$ & [128] \\
\hline P. citrinum & - & C soft coral & Van Phong Bay (Vietnam) & JBIR-27, Petasol, Sporogen AO-1, Dihydrosporogen AO-1 & [129] \\
\hline Penicillium sp. & F011 & sediment & Korea & Herqueiazole, Herqueioxazole, Herqueidiketal & [130] \\
\hline Penicillium sp. & FF001 & S Melophlus sp. & Cicia (Fiji) & Citrinin & [131] \\
\hline $\begin{array}{l}\text { P. pinophilum } \\
\text { (T. pinophilus) }\end{array}$ & SD-272 & sediment & $\begin{array}{l}\text { Pearl River estuary } \\
\text { (China) }\end{array}$ & $\begin{array}{l}\text { Pinodiketopiperazine A, 6,7-Dihydroxy-3-methoxy-3-methyl phthalide, } \\
\text { Cyclo(D-prolyl-D-valyl), Cyclo(trans-4-OH-D-prolyl-D-phenylalanyl), } \\
\text { N-methylphenyldehydroalanyl-L-prolin-anhydrid, L-5-Oxoproline methyl ester, } \\
\text { Rubralide C, Alternariol 2,4-dimethyl ether, Altenuene, 5'-Epialtenuene }\end{array}$ & [132] \\
\hline Penicillium sp. & - & В Fucus spiralis & $\begin{array}{l}\text { Shetland Islands } \\
\text { (Scotland) }\end{array}$ & Patulin, Epiepoformin, Phyllostine, Cladosporin & [133] \\
\hline Penicillium sp. & MWZ14-4 & $\mathbf{S}$ unidentified sponge & $\begin{array}{c}\text { Weizhou } \\
\text { (South China Sea) }\end{array}$ & $\begin{array}{l}\text { Penicimarin A-F, Penicifuran A-D, Aspergillumarin A-B, Sescandelin-B, } \\
\text { 5,6,8-Trihydroxy-4-(1'-hydroxyethyl)isocoumarin }\end{array}$ & [134] \\
\hline Penicillium sp. & SCSIO00258 & C Dichotella gemmacea & Sanya (Hainan, China) & $\begin{array}{l}\text { Penilloid A, Roquefortine C, Isoroquefortine C, Methoxyroquefortine C, } \\
\text { Meleagrin, Glandicoline B, Neoxaline, } \\
\text { (Z)-3-(1H-Imidazole-4-yimethylene)-6-(1H-indl-3-ylmethyl)-2,5-piperazinediol }\end{array}$ & [135] \\
\hline Penicillium sp. & SCSGAF0023 & C D. gemmacea & Sanya (Hainan, China) & $\begin{array}{l}\text { Paecilin C, 6,8,5'6'-Tetrahydroxy-3'-methylflavone, Emodin, Citrorosein, } \\
\text { Isorhodoptilometrin, Penicillixanthone A, Secalonic acid B-D }\end{array}$ & [136] \\
\hline Penicillium sp. & SF-5203 & intertidal sediment & Wan Island (Korea) & Fructigenine A, Cyclopenol & [137] \\
\hline Penicillium sp. & SF-5292 & $\mathrm{Z}$ unidentified Bryozoan & Jeju Island (Korea) & Penicillinolide A, Cycloexpansamine A-B & {$[138,139]$} \\
\hline Penicillium sp. & SF-5295 & S unidentified sponge & Jeju Island (Korea) & Viridicatol & [137] \\
\hline
\end{tabular}


Table 1. Cont

\begin{tabular}{|c|c|c|c|c|c|}
\hline Species Name $^{1}$ & Strain No. & Source of Isolation $^{2}$ & Location & Products $^{3}$ & References \\
\hline $\begin{array}{l}\text { Penicillium sp. } \\
\text { (P. glabrum) }\end{array}$ & JF-55 & S unidentified sponge & Jeju Island (Korea) & Penstyrylpyrone, Anhydrofulvic acid, Citromycetin & [140] \\
\hline Penicillium sp. & JF-72 & S unidentified sponge & Jeju Island (Korea) & $\begin{array}{l}\text { Deoxyisoaustamide, Deoxydihydroisoaustamide, } \\
16 \beta \text {-Hydroxy-17 } \beta \text {-methoxy-deoxydihydroisoaustamide }\end{array}$ & [141] \\
\hline P. chrysogenum & EN-118 & B Sargassum pallidum & Fujian (China) & $\begin{array}{l}\text { Chrysotriazole A-B, 2-(4-Hydroxybenzoyl)-4(3H)-quinazolinone, } \\
\text { 2-(4-Hydroxybenzyl)quinazolin-4(3H)-one), 2-(4-Hydroxyphenyl)acetylamide), } \\
N \text {-(2-(4-Hydroxyphenyl)acetyl)formamide, } N \text {-(2E)-(4-Hydroxyphenyl) } \\
\text { ethenyl)formamide, } N \text {-(2Z)-(4-Hydroxyphenyl)ethenylformamide }\end{array}$ & [142] \\
\hline Penicillium sp. & ZLN29 & sediment & Jiaozhou Bay (China) & $\begin{array}{l}\text { Penicillide, Prenpenicillide, Prenxanthone, Bioxanthracene, NG-011, NG-012, } \\
\text { 15-G256 } \alpha-2,15-\mathrm{G} 256 \beta\end{array}$ & [143] \\
\hline Penicillium sp. & F37 & S A. corrugata & Arvoredo Island (Brazil) & cis-Cyclo(leucyl-tyrosyl) & [144] \\
\hline Penicillium sp. & PR19N-1 = MBC06294 & sludge (depth 1000 m) & Prydz Bay (Antarctica) & $\begin{array}{l}\frac{1 \text {-Chloro-3 } \beta \text {-acetoxy-7-hydroxy-trinoreremophil-1,6,9-trien-8-one, }}{1-\alpha \text {-Chloro-2 } \beta \text {-hydroxyeremophil-7(11),9-dien-8-one, }} \\
\frac{1 \alpha \text {-Chloro-2 } \beta \text {-hydroxyeremophil-7(11),9-dien-8-one, }}{5 \text { new eremophilane compounds, Eremofortine C }}\end{array}$ & {$[145,146]$} \\
\hline P. citrinum & SCSGAF167 & C Echinogorgia aurantiaca & Sanya (Hainan, China) & $\begin{array}{l}\text { Penicitrinol G-H, 2,11-Dihydroxy-1-methoxycarbonyl-9-carboxylxanthone, } \\
\text { Chrysophanol }\end{array}$ & [147] \\
\hline P. brefeldianum & SD-273 & sediment (depth $100 \mathrm{~m}$ ) & $\begin{array}{l}\text { Pearl River estuary } \\
\text { (China) }\end{array}$ & $\begin{array}{l}\text { Verruculogen, 24-Hydroxyverruculogen, 26-Hydroxyverruculogen, } \\
\text { 13-O-Prenyl-26-hydroxyverruculogen, Fumitremorgin A, } \\
\text { Cyclotryprostatin A, TR-2 }\end{array}$ & [148] \\
\hline P. commune & 366606 & water & Qingdao (China) & Penicilliumine & [149] \\
\hline P. echinulatum & pt-4 & R Chondrus ocellatus & Pingtan Island (China) & Arisugacin $C, G, J, \underline{K}$, Territrem $C$ & [150] \\
\hline Penicillium sp. & F446 & sediment (depth 25 m) & $\begin{array}{l}\text { Geomun-do Island } \\
\text { (Korea) }\end{array}$ & Penicillipyrone A-B & [151] \\
\hline T. trachyspermus & KUFA0021 & S Clathria reianwardii & Kram Island (Thailand) & Spiculisporic acid E, Glaucanic acid, Glauconic acid & [152] \\
\hline P. chrysogenum & PJX-17 & sediment & South China Sea & 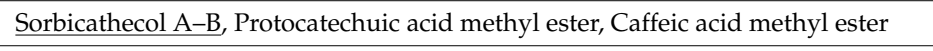 & [153] \\
\hline Penicillium sp. & SF-5995 & C unidentified soft coral & $\begin{array}{l}\text { Terra Nova Bay } \\
\text { (Antarctica) }\end{array}$ & Methylpenicinoline & [154] \\
\hline P. adametzioides & AS-53 & S unidentified sponge & $\begin{array}{c}\text { Wenchang } \\
\text { (Hainan, China) }\end{array}$ & $\begin{array}{l}\text { Lapatin A-B, Prelapatin B, N-Formyllapatin A, Glyantrypine, Adametizine A-B, } \\
\text { Adametacorenol A-B, Peniciadametizine A-B, Brasiliamide A, Viridicatumtoxin, } \\
\text { Fumitremorgin B, Verruculogen }\end{array}$ & [155-157] \\
\hline Penicillium sp. & SF-6013 & U Brisaster latifrons & Sea of Okhotsk (Russia) & Tanzawaic acid A-B,D-E, 2E,4Z-Tanzawaic acid D & [158] \\
\hline P. bialowiezense & IBT28294 & water & North Sea & $\begin{array}{l}\text { Asperphenamates, Mycophenolic acid, F13459, Andrastin A, } \\
\text { Chrysogeside B-E, Quinolactacin A, Raistrick phenols, Xanthoepocin, } \\
\text { Citreohybridonol, Preaustinoids, Fellutamides, Breviones }\end{array}$ & [159] \\
\hline P. lividum & KMM4663 & B Sargassum miyabei & $\begin{array}{l}\text { Lazurnaya Bay } \\
\text { (Sea of Japan) }\end{array}$ & Sargassopenilline B-G & [160] \\
\hline P. thomii & KMM4645 & B S. miyabei & $\begin{array}{l}\text { Lazurnaya Bay } \\
\text { (Sea of Japan) }\end{array}$ & $\underline{\text { Sargassopenilline A,E }}$ & [160] \\
\hline
\end{tabular}


Table 1. Cont

\begin{tabular}{|c|c|c|c|c|c|}
\hline Species Name $^{1}$ & Strain No. & Source of Isolation $^{2}$ & Location & Products $^{3}$ & References \\
\hline Talaromyces sp. & LF458 & S A. verrucosa & Elba Island (Italy) & $\begin{array}{l}\text { Talaromycesone A-B, Talaroxanthenone, Vermixocin A-B, AS-186c, } \\
\Delta 1^{\prime}, 3^{\prime},-1^{\prime} \text {-Dehydroxypenicillide, } 1^{\prime}, 2^{\prime} \text {-Dehydropenicillide, } \\
3^{\prime} \text {-Methoxy-1 } 1^{\prime} 2^{\prime} \text {-dehydropenicillide }\end{array}$ & [161] \\
\hline Talaromyces sp. & SBE-14 & C Subergorgia suberosa & $\begin{array}{c}\text { Weizhou } \\
\text { (South China Sea) }\end{array}$ & $\begin{array}{l}\text { Talaromycin A-C }{ }^{4}, \text { Penicillide, } \\
\Delta 1^{\prime}, 3^{\prime},-1^{\prime} \text {-Dehydroxypenicillide,Purpactin } A, C, C^{\prime}, \text { Tenellic acid methyl esther }\end{array}$ & [162] \\
\hline $\begin{array}{l}\text { P. pinophilum } \\
\text { (T. pinophilus) }\end{array}$ & XS-20090E18 & C unidentified gorgonian & $\begin{array}{c}\text { Xisha Island } \\
\text { (South China Sea) }\end{array}$ & $\begin{array}{l}\text { Purpactin A, Penicillide, Isopenicillide, Hydroxypenicillide, Sch1385568, } \\
\text { Sch725680, Pinophilin B,D-F, Mitorubrin, Mitorubrinol, Mitorubrinic acid }\end{array}$ & [163] \\
\hline T. miniolouteus & PILE14-5 & S unidentified sponge & Phi Phi Island (Thailand) & Minioluteumide A-D, Purpuride, Purpuride B, Berkedrimane B & [164] \\
\hline $\begin{array}{l}\text { P. claviforme } \\
\text { (P. vulpinum) }\end{array}$ & KMM4665 & P Z. marina & $\begin{array}{l}\text { Peter the Great Gulf } \\
\text { (Russia) }\end{array}$ & $\begin{array}{l}\text { 3-[2'(R)-Hydroxybutyl]-7-hydroxyphthalide, } \\
\text { (-)-3-Butyl-7-hydroxyphthalide, Isopatulin, Cyclopenin, Cyclopeptin }\end{array}$ & [165] \\
\hline P. vinaceum & CYE-88 & $\mathbf{S}$ Hyrtios erectus & Yanbu (Saudi Arabia) & $\begin{array}{l}\text { Penicillivinacine, Cyclo(D-tryptophanyl-L-prolyl), Citreoisocoumarin, } \\
\text { Brevianamide F, Indol-3-carbaldehyde, } \alpha \text {-Cyclopiazonic acid, Terretrione A }\end{array}$ & [166] \\
\hline Penicillium sp. & CYE-87 & T Didemnum sp. & Suez Canal (Egypt) & $\begin{array}{l}\text { Terretrione C- } \underline{\mathrm{D}} \text {, Indol-3-carbaldehyde, 3,6-Diisobutylpyrazin-2(1H)-one, } \\
\text { Methyl-2-([2-(1H-indol-3-yl)ethyl]carbamoyl)acetate, Tryptamine }\end{array}$ & [167] \\
\hline Penicillium sp. & IO1 & S Ircinia oros & Kermer (Turkey) & Fusarielin I, Griseofulvin, Dechlorogriseofulvin & [16] \\
\hline Penicillium sp. & IO2 & S I. oros & Kermer (Turkey) & Curvularin, Dehydrocurvularin, Trichodimerol & [16] \\
\hline P. expansum & $\mathrm{Y} 32$ & water & $\begin{array}{l}\text { Indian Ocean, } \\
\text { west of Sumatra }\end{array}$ & $\begin{array}{l}\text { Communesine A-B,I, Fumiquinazoline Q, Prelapatin B, Penochalasin E, } \\
\text { Glyantripine, Protuboxepin A-B,E, Cottoquinazoline A, Chaetoglobosin C }\end{array}$ & [168] \\
\hline Penicillium sp. & KCB12F005 & sediment & Haenam (Korea) & $\underline{\text { Haenamindole }}$ & [169] \\
\hline Penicillium sp. & CF07370 & sediment (depth 100 m) & $\begin{array}{l}\text { Gulf of California } \\
\text { (Mexico) }\end{array}$ & Tanzawaic acid B,E,M-P & {$[170]$} \\
\hline Penicillium sp. & TPU1271 & $\begin{array}{l}\text { organic debris attached to } \\
\text { oyster }\end{array}$ & Oshika Peninsula (Japan) & $\begin{array}{l}\text { Penicyrone A-B, Verrucosidin, Fructigenine A, Verrucofortine, } \\
\text { Cyclo(L-Tryptophanyl-L-Phenylalanyl), Cyclopenol, Cyclopenin, Penipratynolene, } \\
\text { Aspterric acid, Viridicatol }\end{array}$ & [171] \\
\hline P. concentricum & ZLQ-69 & water & Bohai Sea (China) & Phenylpyropene B-D,E-E, Pyripyropene A-B,E,O & [172] \\
\hline $\begin{array}{l}\text { P. verruculosum } \\
\text { (T. verruculosum) }\end{array}$ & TPU1311 & T Polycarpa aurata & Manado (Indonesia) & 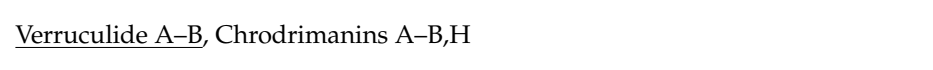 & [173] \\
\hline
\end{tabular}

${ }^{1}$ Current species name is specified in parentheses if different from the one reported in the original reference; ${ }^{2}$ Information concerning the kind of organism is indicated as follows: $\mathbf{B}=$ brown alga; $\mathbf{C}=$ coral, soft coral; $\mathbf{F}=$ fish; $\mathbf{G}=$ green alga; $\mathbf{M}=$ mollusc; $\mathbf{P}=$ Angiosperm plant; $\mathbf{R}=$ red alga; $\mathbf{S}=$ sponge; $\mathbf{T}=$ tunicate; $\mathbf{U}=$ urchin; $\mathbf{Z}=$ bryozoan; ${ }^{3}$ Products originally characterized from the corresponding strain are underlined; ${ }^{4}$ Talaromycins A-C have been reported as new products. However, the same name was previously used for compounds with a different structure isolated from terrestrial Talaromyces strains $[174,175]$ 
Concerning sources, 49 strains were recovered from inanimate substrates, mainly sediment and water samples. As for living organisms, sponges appear to be the most widely reported hosts with 33 strains, confirming recent evidence of their significant interaction with fungi [176,177], while the other sources are represented by a disparate set of animals and plants including shellfish, gorgonians and corals, a few tunicate, urchin and fish species, brown, red and green algae, and a single Angiosperm plant (Zostera marina).

About half of the compounds listed in Table 1 (underlined names) were first characterized in strains from marine sources. This remark not only indicates, once again, that sea is a fruitful context for drug discovery, but also introduces a point of view that the ecological relationships established with marine organisms by species which are ordinarily reported from terrestrial environments may somehow address the biochemical pathways toward the synthesis of some peculiar compounds. In this sense, it must be emphasized that a number of unusual molecular structures have been first elucidated from this biological material (Figures 1-8).

The rest of the compounds itemized in Table 1 were first extracted and characterized for their bioactivity from terrestrial fungal strains, and a few of them are already known as drugs, or drug prospects. Particularly, mycophenolic acid is famous as the first known fungal antibiotic, discovered as a product of a strain of Penicillium brevicompactum even before the start of the 20th century [178], although its real structure was elucidated only after a few decades [179]. This compound displayed notable antibiotic, antiviral, and cytostatic properties, and has found consistent medical application as an immunosuppressive drug in the derivate form of mycophenolate mofetil $[180,181]$. First extracted from a strain of Penicillium griseofulvum [182], but later detected in many congeneric species, griseofulvin gained notoriety as an antimycotic drug, and more recently is being considered for its antitumor properties [183]. Again with reference to their antibiotic/cytotoxic properties, gliotoxin and the chaetoglobosins were first characterized from unrelated fungi, respectively Gliocladium fibriatum [184], and Chaetomium globosum [185]. However, both these compounds were later detected in a few Penicillium species, and considered for a series of interesting effects on human tumor cells [24]. Finally, 3-O-methylfunicone was first identified in connection with the antagonistic/mycoparasitic aptitude of the producing strains of Penicillium pinophilum (=Talaromyces pinophilus) [186,187], and later thoroughly characterized for its cytostatic properties on a number of human tumor cell lines, based on effects on cytoskeletal organization, cell cycle progression, the expression of pro-apoptotic genes, the inhibition of markers of tumor progression, and other mechanisms suppressing cell proliferation/migration [188-196]. Moreover, remarkable activity as a DNA polymerase inhibitor makes it one of the few known natural compounds displaying this particular effect [83]. Taken together, these valuable biological properties introduce 3-O-methylfunicone as a candidate molecule for more accurate clinical investigations in view of its development as an antitumor drug [197].

With a complex structure based on highly oxygenated, bicyclic and tricyclic frameworks, sorbicillinoids are a class of compounds which include over 50 members [198]. Their name derives from sorbicillin, which was first extracted from a terrestrial strain of Penicillium notatum (=Penicillium chrysogenum) [199]. However, a significant number of analog compounds showing peculiar structural models and consistent bioactive properties have been reported from marine fungi. Producing strains cited in this review were ascribed to a few unrelated species, such as Penicillium citrinum, Penicillium crustosum and Penicillium commune (Table 1). However, authorities in Penicillium taxonomy consider these strains to have been probably misidentified, by reason of strict evidence that these products are typical of P. chrysogenum and allied species [200].

Other products listed in Table 1 are best known for their noxious effects as mycotoxins contaminating foodstuffs. This is the case of cyclopiazonic acid, verrucosidin, fumitremorgin, and a few related tremorgenic toxins, secalonic acids, and particularly of citrinin and patulin [25]. Actually, the concern for dietary intake of mycotoxins produced by Penicillium strains has recently reached seafood, and specific investigations are being carried out in order to better assess the associated risk for consumers [54,128,201]. 
<smiles>CC(=O)C1OC(=O)c2c(O)cc(O)cc21</smiles>

Acetophthalidin<smiles>[R]c1ccccc1-n1c([C@@H]2CC[C@H]([NH3+])N2)nc2ccccc2c1=O</smiles>

Auranomides

Auranomide $\mathrm{AR}=\mathrm{COO}$ Auranomide $\mathrm{BR}=\mathrm{COOCH}_{3}$<smiles>CC(=O)C[C@H]1CCC2=CC(=O)C(=C(C)C)[C@H](O)[C@]2(C)[C@@H]1C</smiles>

3-Acetyl-9,7(11)-dien-7 $\alpha$-hydroxy8-Oxoeremophilane<smiles>NC(=O)CC[C@@H]1NC(=O)c2ccccc2-n2c1nc1ccccc1c2=O</smiles>

Auranomide $\mathrm{C}$<smiles>C=C(C)[C@H]1[C@@H](CC(C)=O)C[C@H](C)C12CC=C(CO)[C@@H](O)C2</smiles>

Adametacorenol B<smiles>[R][C@]1(CC(C)C)NC(=O)[C@H](CCC(N)=O)n2c1nc1ccccc1c2=O</smiles>

Aurantiomide $\mathrm{BR}=\mathrm{OH}$ Aurantiomide $\mathrm{C} A B=\triangle$<smiles>[R]C1C=C(C)C2(CC3(C)Cc4c(c(C)c(C)oc4=O)O3)[C@@H](CCC1C1(C)C=CC(=O)C(C)(C)C1)[C@H]2C</smiles><smiles>[R]C1C=C(C)C2(CC3(C)CCC4C(C)=CC(=O)C=CC4(C)[C@H]32)Oc2c1c(C)c(C)oc2=O</smiles>

Breviones, type structures

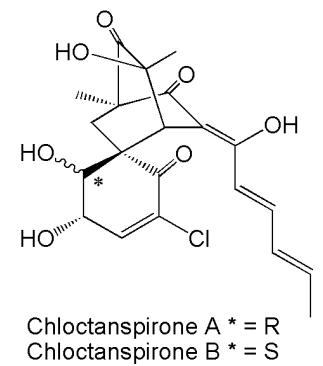<smiles>CC(=O)O[C@H]1C=C(Cl)C2=CC(=O)C(O)=C[C@]2(C)[C@H]1C</smiles>
1-Chloro-3 3 -acetoxy-7-hydroxy- Citrinadin $A \mathrm{R}=\mathrm{y}_{2}$
trinoreremophil-1,6,9-trien-8-one<smiles>[R]C1C[C@H](C)N2C[C@]3(NC)C[C@]4(C(=O)Nc5c(C(=O)C6OC6(C)C)cccc54)[C@@](C)(O)[C@@]3(O)C[C@H]2C1</smiles><smiles>CC1=C2[C@]3(O)CC(=O)[C@@]2(O[C@H](C)[C@@H](C)O3)[C@@H](C)[C@@H]1C</smiles>

Citrinal A

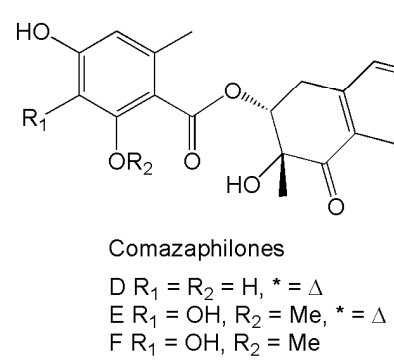

$D \mathrm{R}_{1}=\mathrm{R}_{2}=\mathrm{H}^{*}{ }^{*}=$ $\mathrm{FR}_{1}=\mathrm{OH}, \mathrm{R}_{2}=\mathrm{Me}$

Brocaenol A Brocaenol B R $=\mathrm{H}$ Brocaenol $\mathrm{CR}=\mathrm{CH}_{3}$

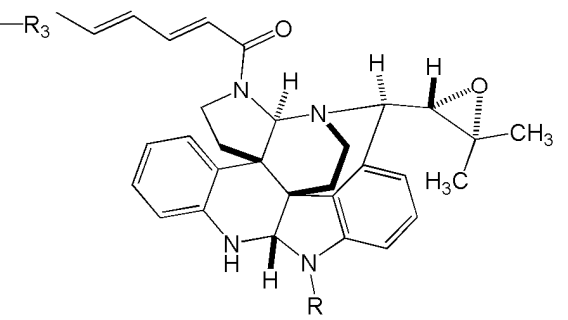

Communesins, general formula

Figure 1. Cont. 


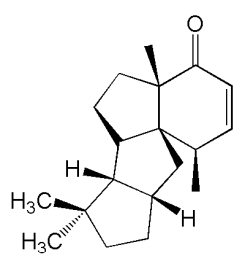

Conidiogenone B<smiles>C/C=C/C=C/C(=O)c1c(O)c(C)cc(C)c1O</smiles>

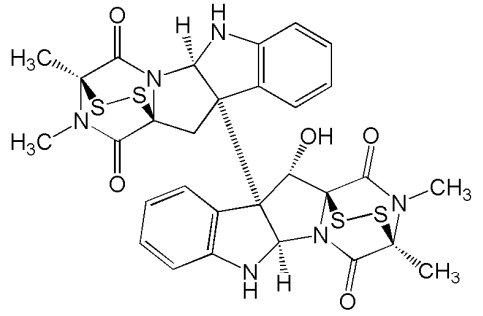

11'-Deoxyverticillin A type structure

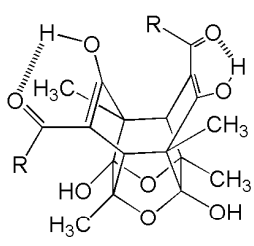

Dihydro and tetrahydrorrichodimerol type structures $\mathrm{R}=\mathrm{C}_{5} \mathrm{H}_{9}$ and or $\mathrm{C}_{5} \mathrm{H}_{7}$<smiles>[R]C1([R2])C(=O)Nc2ccccc2[C@@]1(O)c1ccc(OC)cc1</smiles>

(2E,4E)-1-(2,6-Dihydroxy-3,5-dimethylphenyl)hexa-2,4-dien-1-one

$3 S^{*}, 4 R^{*}$-Dihydroxy-4-(4'-methoxyphenyl)3,4-dihydro-2(1H)-quinolinone

Epolactaene $\mathrm{R}_{1}=\mathrm{HR}_{2}=\mathrm{OH}$<smiles>[Y]C(O)[C@H](O)CC(=O)N[C@@H](CC(N)=O)C(=O)N[C@H](C(=O)N[C@@H](C=O)CC(C)C)[C@]([R])([2H])CC(N)=O</smiles><smiles>COC1=CC(=O)C(C)=C(CC(C)CC=O)C1=O</smiles><smiles>COC1=CC(=O)[C@]2(C)O[C@]23CC(C)O[C@]13O</smiles>
$\overbrace{\mathrm{NH}_{2}}^{\mathrm{R}}$

Fellutamide $\mathrm{AR}=\mathrm{OH}$

(+)-Formylanserinone B

Epoxyserinone B Fellutamide BR $=\mathrm{H}$<smiles>C=C1C(=O)N2[C@@H](CC3=CC=C[C@H](O)[C@@H]32)CC1(C)C</smiles>

Bis(dethio)-10a-methylthio-3a-deoxy3,3a-didehydrogliotoxin type structure

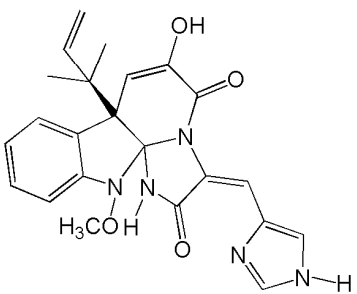

Meleagrin type structure<smiles>COc1c(O)c2c(c3c1OC1(O)O[C@H](C)C(C)(C)[C@@]1(O)O3)CC(C)=CC2=O</smiles>

Herqueidiketal<smiles>[R]C1C[C@H]2C(C)(C)CC[C@@H](OC(=O)C(NC(C)C)C(C)C)[C@@]2(C)C2=C1C(=O)OC2</smiles>

Minioluteumides type structures<smiles></smiles>

JBIR-28

Penicacid A

Figure 1. Cont. 


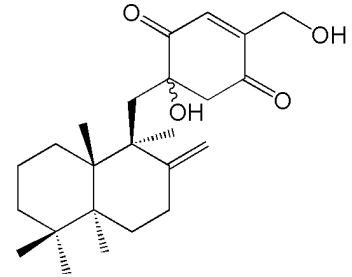

Penicilliumin A

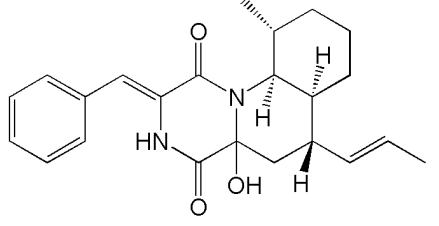

Penicillivinacine

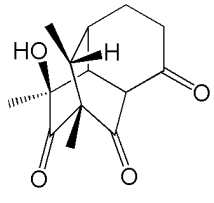

Penicillone A

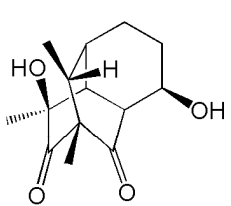

Penicillone B<smiles>CC(CCCC[C@H]1C[C@@H](O)CC(=O)O1)CC(C)CC[C@@H](C)O</smiles>

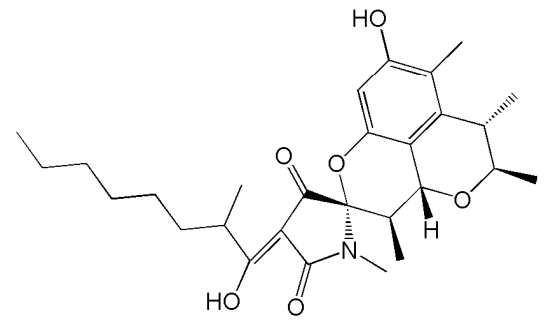

Penicitrinine A<smiles>CC(=O)CC1OC(C)C(C)c2c(O)cc(O)c(C)c21</smiles>

Penicitrinol C<smiles>CO[C@]1(C)C[C@H]2O[C@H](C)[C@@H](C)c3c(cc(O)c(C)c32)O1</smiles>

Penicitrinol E<smiles>C/C(=N\CC(C)(C)O)Nc1ccccc1C(=O)O</smiles>

Penipacid A<smiles>C/C(=C\Cc1cccc2c(C=O)c[nH]c12)CO</smiles>

Penipalin C<smiles>COC(=O)/N=C(\C)Nc1ccccc1C(=O)O</smiles>

Penipacid E<smiles>O=C(O)c1ccccc1-n1ncnc1Cc1ccc(O)cc1</smiles>

Penipanoid A<smiles>CC(C)=CCc1cccc2c3c([nH]c12)C(C)(C)N[C@H](C(=O)O)C3</smiles>

Penipalin B

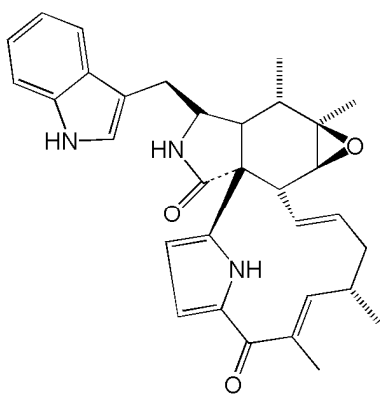

Penochalasin D type structure

Figure 1. Cont. 


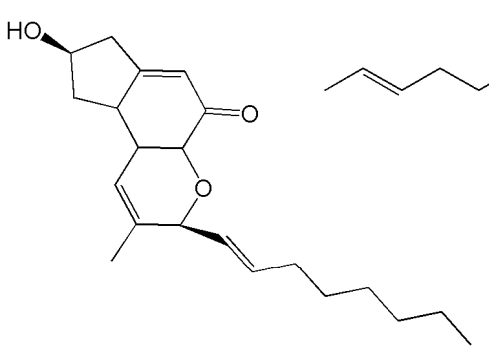

Penostatin A type structure

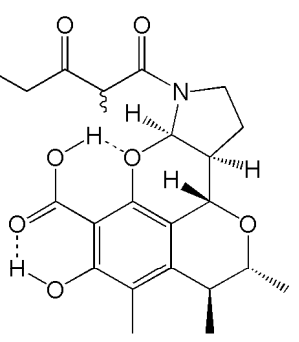

Perinadine A

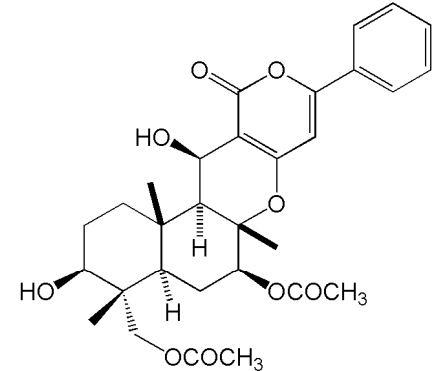

Phenylpyropene E

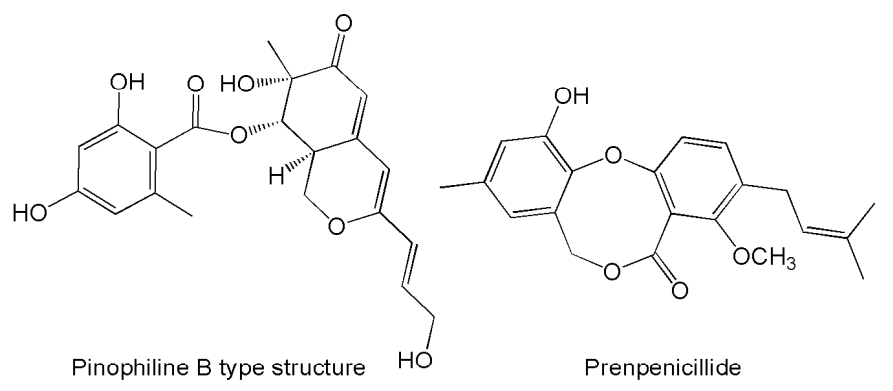<smiles>COc1cc(=O)oc(C)c1C(=O)CC(C)OC</smiles>

Pyrenocine E<smiles>CC1C[C@H](C)CC2(CC3=C(CO2)C(=O)C(C)(O)[C@@H](O)C3)O1</smiles>

Sargassopenilline E<smiles>CC1(C)C=C2c3cc4[nH]c5c(c4cc3C(O)C2(C)C(C)(C)O1)CC1CC[C@]2(O)C3=CC(=O)C4OC3(CC[C@@]2(C)[C@]54C)O1</smiles>

Shearinine $\mathrm{E},{ }^{*} \mathrm{H}_{22}, \mathrm{H}_{23}$ cis type structure<smiles>C/C=C/C=C/C(O)=C1/C(=O)C(C)=C(O)[C@@]2(C)OC(=O)[C@](C)(NC(=O)/C=C/C(=O)O)[C@]12C</smiles>

Sorbicillactone A

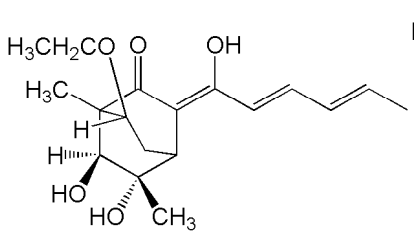

Sorbivinetol

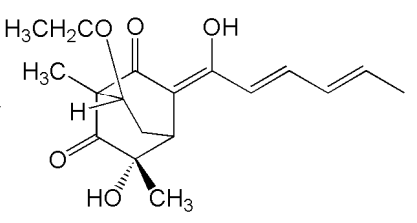

Sorbivinetone

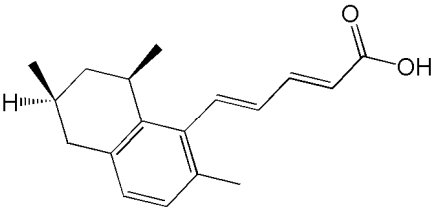

Tanzawaic acid A type structure<smiles>COCc1cc(O)c(Cc2cc(O)c(Cc3cc(O)ccc3O)cc2O)cc1O</smiles>

Terrestrol A type structure

Terretrione D

Figure 1. Structures of novel compounds produced by marine Penicillium/Talaromyces strains displaying inhibitory properties against mammalian tumor cell lines. For the sake of space, compounds produced in series of two or more analogs are presented as a single or type structure. 
<smiles>[R]c1cc2c3c(c(O)c(OC)c(O)c3c1C)C(=O)C(O)(CC(C)=O)C2=O</smiles>

Sculezonone $\mathrm{AR}=\mathrm{H}$ Sculezonone $\mathrm{BR}=\mathrm{OH}$<smiles>[R3]CC([R3])CCCC[C@H](C(=C)C(=O)O[R])C(=O)O[R]</smiles>

Oxygenated hexylitaconates

a. $\mathrm{R}_{1}=\mathrm{R}_{2}=\mathrm{Me} \mathrm{R} \mathrm{R}_{3}=\mathrm{OH} \mathrm{R}_{4}=\mathrm{H}$

b. $\mathrm{R}_{1}=\mathrm{R}_{2}=\mathrm{H}=\mathrm{R}_{3}=\mathrm{OH} \mathrm{R}_{4}=\mathrm{H}$

c. $R_{1}=R_{2}=R_{3}=H R_{4}=O H$

d. $R_{1}=R_{2}=M e R_{3}=H R_{4}=O H$

(3S)-Hexylitaconic acid $\mathrm{R}_{1}=\mathrm{R}_{2}=\mathrm{R}_{3}=\mathrm{R}_{4}=\mathrm{H}$<smiles>CC(=O)OC1C2=C(CC(=O)C1(C)OC(C)=O)C(=O)C(=O)CC21OC[C@H](C)C[C@H]1O</smiles>

Sargassopenillin C<smiles>C[C@H]1Cc2c3c(cc(O)c2C(=O)O1)O[C@]1(C)CC[C@]2(C)C(C)(C)C(=O)C=C[C@]2(C)[C@]1(C)C3</smiles>

Verruculide A

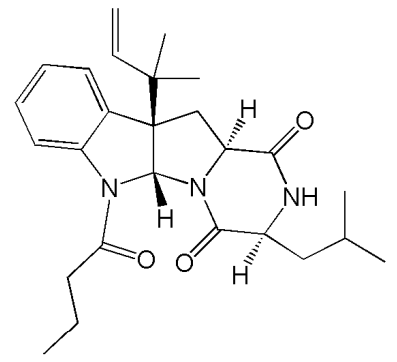

Brevicompanin E

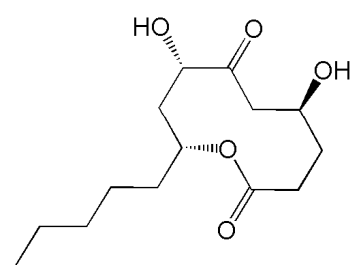

Penicillinolide A<smiles>COc1cc(/C=C/c2ccccc2)oc(=O)c1C</smiles>

Penstyrylpyrone

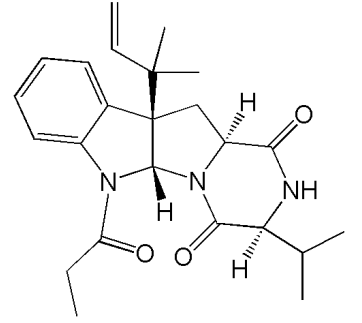

Brevicompanin $\mathrm{H}$<smiles>CC(=O)c1cc(C)c(O)c(CC23Cc4c(C(C)=O)cc(C)c(O)c4OC2(O)C(C)OC3=O)c1O</smiles>

Penilactone A<smiles>OCc1cc(O)c(Cc2cc(O)ccc2O)c(Cl)c1O</smiles>

Terrestrol G

Figure 2. Structures of novel compounds produced by marine Penicillium/Talaromyces strains displaying anti-inflammatory effects. 
<smiles>COc1cccc2c(=O)c(C)c(CO)oc12</smiles>

Chromanone A

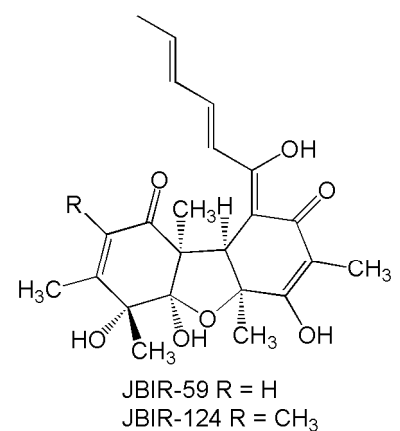<smiles>[R7]c1c(O)cc(Oc2c([R])c(C)c([R])c(O)c2[R])c([R])c1C</smiles>

Polybrominated diphenyl ethers $\mathrm{R}_{1}$ and/or $\mathrm{R}_{2}=\mathrm{Br}$<smiles>[R]c1cccc2c(/C=C3\NC(=O)[C@@H](C)NC3=O)c(C(C)(C)C=C)[nH]c12</smiles>

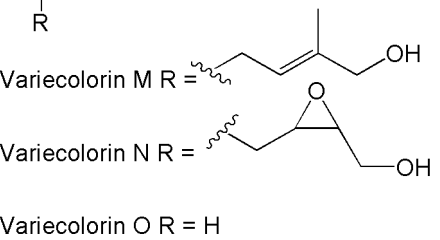<smiles>Cc1c(O)cc2c(c1O)CO[C@@]1(O2)O[C@H](C)CC[C@@H]1O</smiles>

Sargassopenilline A type structure<smiles>Cc1cc(=O)c2c(o1)OC1C(CC2)CCC2C(C)(C)C(=O)CC[C@]12C</smiles>

Penicillipyrone B

Figure 3. Structures of novel compounds produced by marine Penicillium/Talaromyces strains reported for ROS-scavenging properties.

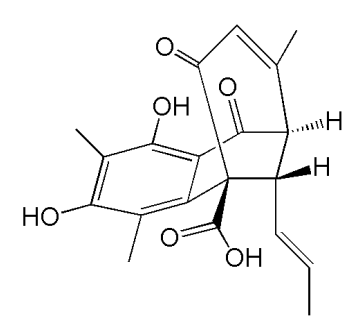<smiles>CC(O)(C(=O)Nc1ccccc1C(N)=O)[C@@](C)(O)c1nc2ccccc2c(=O)[nH]1</smiles>

Sorbiterrin A<smiles>CC(C)=CCc1ccc2oc3cc(C)cc(CO)c3c(=O)c2c1O</smiles>

Talaroxanthenone
Penicilliumine<smiles>NCCCCNC(=O)C(Cc1ccc(O)cc1)NC(=O)C1OC1C(=O)O</smiles>

Cathestatin B

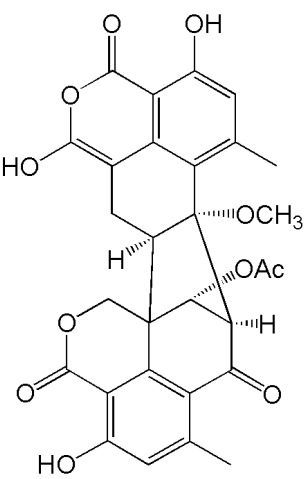

Talaromycesone A

Figure 4. Structures of novel compounds produced by marine Penicillium/Talaromyces strains with enzyme-modulatory activities. 


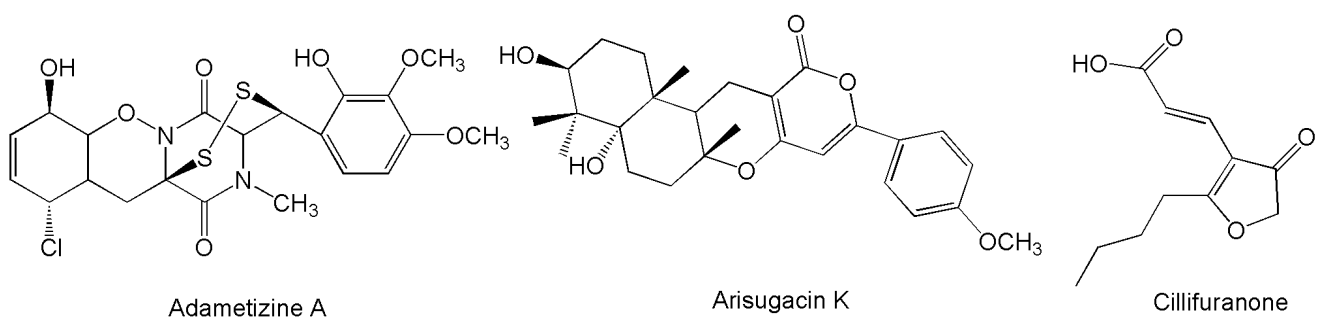<smiles>[R20]c1c(O)cc(C)c(C(=O)O[C@]2(C)C(=O)C3=C(C=C(CC=CC)OC3)C[C@H](O)[C@@]2(C)OC(=O)c2c(C)cc(O)c([R1])c2[R20])c1[R1]</smiles>

Comazaphilones

$$
\begin{aligned}
& A R_{1}=H, R_{2}=M e \\
& \mathrm{BR}_{1}=O H, R_{2}=M e \\
& C R_{1}=O H, R_{2}=M e,{ }^{*}=\Delta
\end{aligned}
$$

$$
\begin{aligned}
& D R_{1}=R_{2}=H,{ }^{*}=\Delta \\
& E R_{1}=O H, R_{2}=M e,{ }^{*}=\Delta \\
& F R_{1}=O H, R_{2}=M e
\end{aligned}
$$<smiles>CC(=O)c1cc(C)c(O)c(Cc2c(O)cc(C)oc2=O)c1O</smiles>

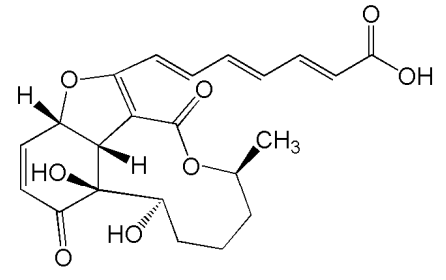

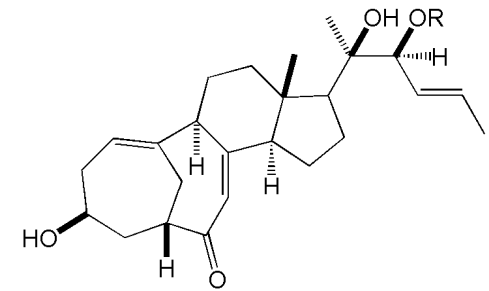

Communol A, type structure

Dictyosphaeric acid A

Isocyclocitrinol $\mathrm{A} \mathrm{R}=\mathrm{H}$ 22-Acetyl isocyclocitrinol A R $=\mathrm{COCH}_{3}$<smiles>COc1ccc2c(c1OC)O[C@]1(C(=O)N3O[C@@H]4C(=CC=C[C@H]4O)C[C@]3(SC)C(=O)N1C)[C@H]2SC</smiles>

Peniciadametizine A<smiles>COC1=CC=C2C=C(C(=O)N3O[C@@]4(C[C@]5(O)[C@@H](O)C=C[C@H](O)[C@]5(SC)C4=O)C(=O)N3C)C(=C2O)OC1OC</smiles>

Peniciadametizine B<smiles>COc1cc(O)cc2c(CO)c(C)oc12</smiles>

Penicifuran A<smiles>O=C1CC(C(=O)CCO)CO1</smiles><smiles>CC(=O)OC/C(C)=C/CCC(C)(O)CC(=O)O</smiles><smiles>CC1=CC(=O)[C@]2(CC[C@@H](C)C(=O)O2)[C@H](O)[C@@H]1O</smiles>

Penicilactone

Penicimonoterpene

Penicyclone A type structure<smiles>CN1C(=O)NC(CCCO)(CCCO)C1=O</smiles>

Pinodiketopiperazine A<smiles>C/C=C/CCCCC(=O)[C@H](C)C(=O)N1CCC[C@H]1CO</smiles>

Scalusamide A<smiles>C[C@@H]1CC(O)CC(=O)c2c(O)cc(O)cc2CC1=O</smiles>

Xestodecalactone B

Figure 5. Structures of novel antibiotic compounds produced by marine Penicillium/Talaromyces strains. 


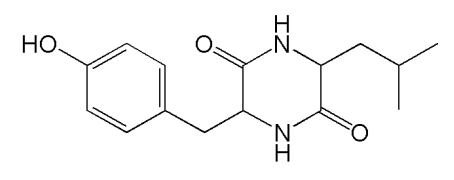

cis-cyclo(Leucyl-Tyrosyl) dipeptide

Figure 6. Structure of cis-cyclo(leucyl-tyrosyl) dipeptide.

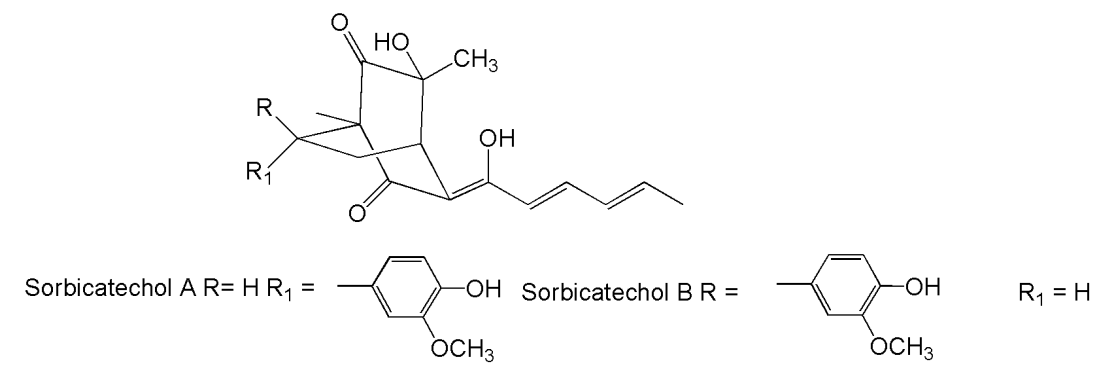

Figure 7. Structures of sorbicathecols.

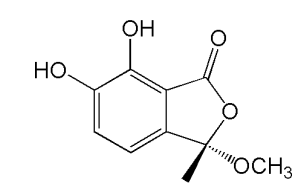

6,7-Dihydroxy-3-methoxy3-methylphthalide

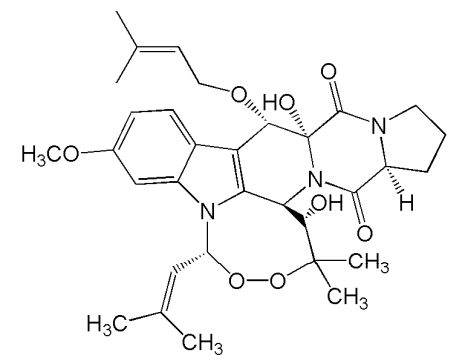

13-O-Prenyl-26-hydroxyverruculogen

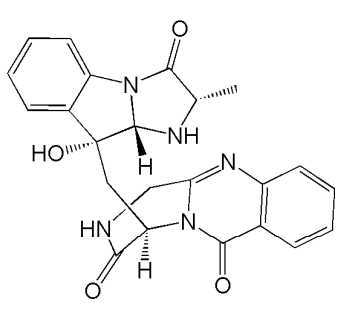

Fumiquinazoline $Q$<smiles>CCC(C)c1nc2c(O)cccc2c(=O)n1[C@@H](Cc1ccccc1)C(=O)N[C@@H](C)CC</smiles><smiles>Cc1cc(O)c(O)c(-c2cc(=O)c3cc(O)cc(O)c3o2)c1</smiles><smiles>COC(=O)c1c(Oc2c(O)cc(C)cc2C(OC)OC)ccc(C(CC(C)C)OC(C)=O)c1OC</smiles>

6,8,5'6'-Tetrahydroxy-3'-methylflavone

Figure 8. Structures of novel compounds produced by marine Penicillium/Talaromyces characterized for miscellaneous bioactive effects.

\section{Bioactivities of Novel Compounds}

By reason of the quite short time having elapsed after their discovery, most of the novel compounds obtained from marine strains of Penicillium/Talaromyces have been characterized for their biological properties and mechanisms of action only at a preliminary stage. In this regard, the largest category of bioactivity is undoubtedly represented by the cytotoxic/antiproliferative products (Table 2, Figure 1). In fact, assays on human or mammalian cell lines have become widespread following the recent trend to identify new natural antitumor compounds [202], and in view of pursuing this general aim there is a tendency to inappropriately consider these terms as synonyms [203]. Even if such a frequent semantic impropriety does not affect the significance of preliminary bioactivity screenings, the possible relevance of these molecules as antitumor prospects can be introduced only when a further characterization of their cytological effects is accomplished, which quite notably reduces the number of compounds deserving to be further examined in this review. 
Table 2. Novel compounds produced by marine Penicillium/Talaromyces strains displaying inhibitory properties against mammalian tumor cell lines.

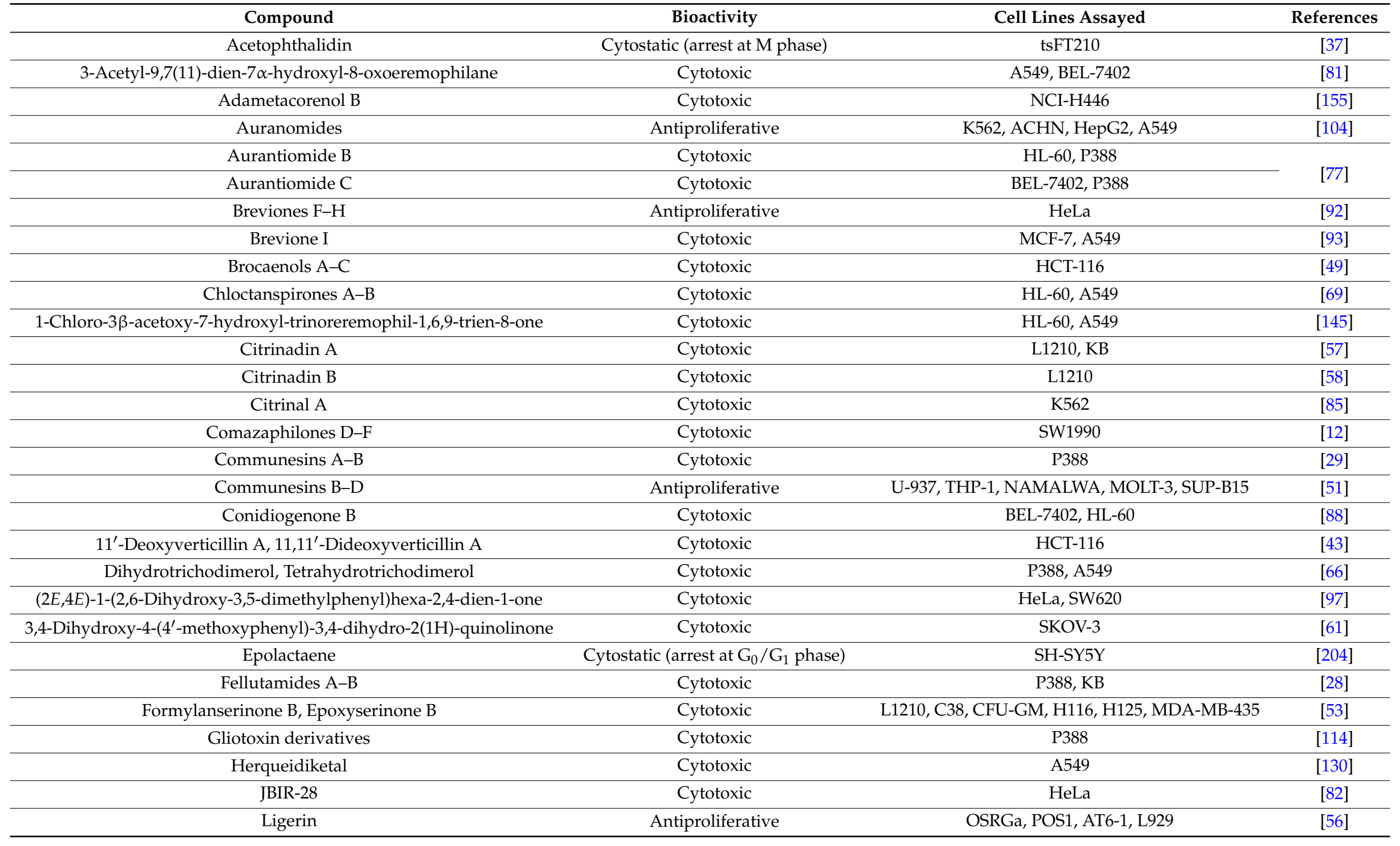


Table 2. Cont.

\begin{tabular}{|c|c|c|c|}
\hline Compound & Bioactivity & Cell Lines Assayed & References \\
\hline \multirow{2}{*}{ Meleagrin B } & Cytotoxic & BEL-7402, HL-60, A549, MOLT-4 & [88] \\
\hline & Pro-apoptotic & HL-60 & [89] \\
\hline Meleagrins D-E & Cytotoxic & A549 & [89] \\
\hline Penicacid A & Antiproliferative & Mouse splenocytes & [124] \\
\hline Penicilliumin A & Cytotoxic & A375, B16, HeLa & [9] \\
\hline Penicillivinacine & Antimigratory & MDA-MB-231 & [166] \\
\hline Penicillone A & Cytotoxic & SW620 & [97] \\
\hline Penicisteroid A & Cytotoxic & HeLa, SW1990, NCI-H460 & [108] \\
\hline Penicitide A & Cytotoxic & HepG2 & [107] \\
\hline Penicitrinine A & Antiproliferative, pro-apoptotic & 23 tumor cell lines & [111] \\
\hline Penicitrinols C, E & Cytotoxic & HL-60 & [112] \\
\hline Penipacids A, E & Cytotoxic & RKO & [117] \\
\hline Penipalines B-C & Cytotoxic & A549, HCT-116 & [118] \\
\hline Penostatins A-C,E-I & Cytotoxic & P388 & [31-33] \\
\hline Perinadine A & Cytotoxic & L1210 & [59] \\
\hline Phenylpyropene E & Cytotoxic & MGC-803 & [171] \\
\hline Pinophilins & Antiproliferative & A549, BALL-1, HCT116, HeLa, NUGC-3 & [122] \\
\hline Prenpenicillide & Cytotoxic & HepG2 & [143] \\
\hline Pyrenocine E & Cytotoxic & P388 & [40] \\
\hline Sargassopenilline E & Cytotoxic & CD-1 & [160] \\
\hline Shearinines & Pro-apoptotic & HL-60 & [74] \\
\hline Sorbicillactones, Sorbivinetol, Sorbivinetone & Cytotoxic & L5178y & [62] \\
\hline Tanzawaic acids & Antiproliferative, pro-apoptotic & K562, U937, Jurkat, Raji & [170] \\
\hline Terrestrols A-H & Cytotoxic & BEL-7402, HL-60, A549, MOLT-4 & [64] \\
\hline Terretrione D & Antimigratory & MDA-MB-231 & [167] \\
\hline
\end{tabular}


Besides consistent pro-apoptotic effects on human promyelocytic leukemia cells, the indole diterpenoid alkaloid shearinine $\mathrm{E}$ has been characterized for its ability to inhibit the malignant transformation of mouse epidermal cells $(\mathrm{JB} 6 \mathrm{P}+\mathrm{Cl} 41)$ experimentally induced by the epidermal growth factor in the anchorage-independent transformation assay [74].

Another alkaloid with a unique spiro skeleton, penicitrinine A, was found to induce some typical modifications in melanoma cells undergoing apoptosis, such as shrinkage, fragmentation, and chromatin condensation. Assays based on annexin-V/PI double staining showed that apoptosis occurred at a higher rate than control cells treated with the chemotherapeutic drug 5-fluorouracile. Apoptosis followed the mitochondrial pathway, as indicated by down-regulation of the anti-apoptotic gene Bcl-2 and concomitant up-regulation of the pro-apoptotic gene Bax, and the ratio of Bcl-2/Bax expression, which decreased with increasing concentrations of the compound. Anti-metastatic dose-dependent effects were also observed as a result of suppression of invasiveness and inhibition of cell migration, which is an ill-fated tendency of melanoma cells. These latter effects are related to a down-regulation of matrix metalloproteinase (MMP-9) expression along with up-regulation of its inhibitor glycoprotein TIMP-1 [111].

Besides selectively suppressing cell growth and proliferation in five human cancer cell lines, pinophilins displayed a strong inhibitory activity on DNA polymerases of the A-, B-, and Y-families, particularly against DNA pol- $\alpha$ and $-\kappa$. The inhibitory effect was selective, since it was not observed on normal human cells (dermal fibroblasts and umbilical vein endothelial cells), possibly because their DNA replication rates are significantly slower than those of cancer cells [122]. Bioactivity as DNA polymerase inhibitors had been previously showed for two phenalenone compounds, the sculezonones A-B. Particularly, both compounds inhibited bovine DNA pol- $\alpha$ and $-\gamma$, and moderately affected the activity of DNA pol- $\varepsilon$. Moreover, DNA pol- $\beta$ was inhibited by sculezonone A, and just weakly influenced by sculezonone B [205]. Another DNA polymerase inhibitor, the $\gamma$-lactam compound epolactaene [36], not only was effective on bovine DNA pol- $\alpha$ and rat DNA pol- $\beta$, but also disclosed inhibitory properties against human DNA topoisomerase II [206], which is a very important biomolecular mechanism considered in prospecting for antitumor drugs [207]. Consistent neuritogenic effects [36] make this compound even more valuable in view of possible medical applications.

Some degree of neural stimulation was also pointed out for the fellutamides. In fact these tripeptides were characterized as potent enhancers of the release of the nerve growth factor (NGF) from fibroblasts and glial-derived cells [208]. This effect results from the inhibition of proteasome catalytic activity, which leads to increased NGF gene transcription [209]. For different respects, neuroprotective properties have been reported for sorbicillactone A, which impaired the negative effects of important neurotransmitters such as L-glutamic acid and serotonin [62], and brevicompanines $\mathrm{E}$ and $\mathrm{H}$ (Figure 2), which have been characterized as neuroinflammation modulators [87]. More in detail, in BV2 mouse microglial cells brevicompanine $\mathrm{E}$ was found to inhibit production of tumor necrosis factor- $\alpha$ (TNF- $\alpha$ ), interleukin-1 $\beta$ (IL-1 $\beta$ ), inducible nitric oxides (iNOS), and cyclooxygenase- 2 (COX-2), and to reduce the DNA binding activity of the oncogenic nuclear factors AP-1 and NF- $\mathrm{KB}$. Nuclear translocation of the latter was also inhibited, together with IKB $\alpha$ degradation, and Akt and c-Jun $\mathrm{NH}_{2}$-terminal kinase phosphorylation [210]. Similar anti-inflammatory effects were also evidenced in murine peritoneal macrophages for novel ester derivatives of hexylitaconic acid [115], and for penicillinolide A [138] (Figure 2). Weak NF-kB inhibitory properties were again reported from penilactone A [125], while sargassopenilline $C$ has been found to inhibit the transcriptional activity of AP-1 [160] (Figure 2). Finally, and again in BV2 cells, 2E,4Z-tanzawaic acid D was found to inhibit the production of iNOS [158].

Penstyrylpyrone (Figure 2) is another product reported for considerable anti-inflammatory activity deriving from inhibition of the expression of iNOS and COX-2, reduction of TNF- $\alpha$ and IL-1 $\beta$ production, suppression of phosphorylation and degradation of I $\kappa B-\alpha$, and of NF- $k B$ nuclear translocation and DNA binding activity. These effects were found to be associated with the expression of heme oxygenase 1 (HO-1), an enzyme releasing anti-inflammatory degradation products during 
heme catabolism. Ultimately, these anti-inflammatory effects lead to a competitive inhibition of the protein tyrosine phosphatase $1 \mathrm{~B}$ (PTP1B), which is known to play a major role in the negative regulation of insulin signalling. Therefore, this compound was introduced as a prospect therapeutic drug for the treatment of type II diabetes [140]. Inhibitory properties towards PTP1B were also disclosed for penostatins A-C [211], while verruculides A and B respectively displayed a strong and a moderate effect against this enzyme [173]. Finally, a moderate effect as an inhibitor of tyrosine kinases was reported for terrestrol G (Figure 2) [64].

Another target in the search for antitumor products is represented by the reactive oxygen and nitrogen species (ROS), whose excessive production results in oxidative stresses, DNA damage, and inflammation, as well as contributing to tumor initiation and promotion. Consequently, scavenging of the physiologically relevant ROS represents an effective strategy in preventing tumor initiation and promotion. Chromanone A (Figure 3) was characterized as a strong $\mathrm{OH}$ scavenger, which also dramatically inhibits the degree of DNA fragmentation. Moreover, it was able to act, in a dose-dependent manner, as a potent inhibitor of cytochrome P4501A, and as an inducer of GSH (cytosolic thiol) and GST enzymes, which both help in the destruction of peroxides, free radicals, and other xenobiotics [86].

Radical scavenging effects were also reported for compound JBIR-59 (Figure 3), on account of its protective effects against L-glutamate toxicity in neuronal hybridoma N18-RE-105 cells [99]. A few more products (Figure 3) have been characterized as free radical scavengers based on their activity against 1,1-diphenyl-2-picrylhydrazyl (DPPH), such as the terrestrols [64], 4,6,4', $6^{\prime}$-tetrabromo-3,3'-dihydroxy-5,5'-dimethyldiphenyl ether and 4,6,2', $4^{\prime}, 6^{\prime}$-pentabromo-3,3'dihydroxy-5,5'-dimethyldiphenyl ether [105], the variecolorins [102], compound JBIR-124 [100], and sargassopenillines A and E [160]. Further indirect antitumor effects resulting from detoxification of xenobiotics have been proposed for the meroterpenoid penicillipyrone B (Figure 3 ) for its ability to induce the enzyme quinone-reductase, which is involved in the reduction of electrophilic quinones [151].

Other enzyme modulatory activities are relevant in human medicine for the treatment of a number of complex diseases. This is the case for Alzheimer's disease, where compounds performing acetylcholinesterase inhibition can be considered in view of possible therapeutic use [212]. In this regard, moderate activity has been reported for products such as sorbiterrin A [65], and penicilliumine [149], while more potent effects have been evidenced for talaromycesone A and talaroxanthenone [161] (Figure 4). The latter compound also showed activity as an inhibitor of phosphodiesterase, which is a target in the treatment of inflammatory processes involved in pulmonary diseases [161]. Besides general activity as inhibitors of proteases, such as papain, ficin, and bromelain, the cathestatins, particularly cathestatin B (Figure 4), were introduced for possible useful effects in the treatment of osteoporosis deriving from the inhibition of bone collagen degradation, and the suppression of calcium release [35]. Finally, along the lines of their more famous analog mycophenolic acid, the penicacids (Figure 4) were investigated for their immunosuppressive properties, and found to possess appreciable inhibitory effects towards inosine-monophosphate dehydrogenase [124].

Antibiotic properties have been assessed for a number of novel compounds (Figure 5) against the bacterial and fungal species indicated in Table 3. Besides these general inhibitory effects, some peculiar mechanisms of action were evidenced for the dipeptide cis-cyclo(leucyl-tyrosyl) (Figure 6), which inhibits biofilm formation by Staphylococcus epidermidis without interfering with bacterial growth [144], and herqueidiketal, which was characterized for inhibitory properties against sortase A of Staphylococcus aureus. Since sortases are absent in mammals, this biochemical effect may be relevant for the development of novel antibiotics [130]. Moreover, the above-mentioned proteasome inhibitory effects by fellutamide B were again observed on Mycobacterium tubercolosis, introducing this peptide as a prospect drug to be more thoroughly investigated against such a deadly pathogen [213]. 
Table 3. Novel antibiotic compounds produced by marine Penicillium/Talaromyces strains.

\begin{tabular}{|c|c|c|c|}
\hline Compound & Bioactivity & Microbial Species Assayed & References \\
\hline \multirow{2}{*}{ Adametizine A } & Antibacterial & Aeromonas hydrophila, Staphyloccocus aureus, Vibrio harveyi, Vibrio parahaemolyticus & \multirow{2}{*}{ [155] } \\
\hline & Antifungal & Gaeumannomyces graminis & \\
\hline Arisugacin K & Antibacterial & Escherichia coli & [150] \\
\hline \multirow{2}{*}{ Cillifuranone } & Antibacterial & Xanthomonas campestris & \multirow{2}{*}{ [98] } \\
\hline & Antifungal & Septoria tritici & \\
\hline Comazaphilones & Antibacterial & Bacillus subtilis, Pseudomonas fluorescens, S. aureus m.r. & [12] \\
\hline Communol A, F-G & Antibacterial & Enterobacter aerogenes, E. coli & [127] \\
\hline \multirow{2}{*}{ Conidiogenone B } & Antibacterial & Pseudomonas aeruginosa, Pseudomonas fluorescens, S. aureus m.r., Staphylococcus epidermidis & \multirow{2}{*}{ [109] } \\
\hline & Antifungal & Candida albicans & \\
\hline \multirow{2}{*}{ Dictyosphaeric acid A } & Antibacterial & Enterococcus faecium, S. aureus, S. aureus m.r. & \multirow{2}{*}{ [50] } \\
\hline & Antifungal & C. albicans & \\
\hline Isocyclocitrinols & Antibacterial & Enterococcus durans, S. epidermidis & [48] \\
\hline Peniciadametizines & Antifungal & Alternaria brassicae & [156] \\
\hline Penicifuran A & Antibacterial & Bacillus cereus, Staphylococcus albus & [134] \\
\hline Penicilactone & Antibacterial & S. aureus m.r. & [95] \\
\hline \multirow{2}{*}{ Penicimonoterpene } & Antifungal & A. brassicae, Aspergillus niger, Fusarium graminearum & {$[107,214]$} \\
\hline & Antibacterial & A. hydrophila, E. coli, Micrococcus luteus, S. aureus, V. harveyi, V. parahaemolyticus & [214] \\
\hline Penicisteroid A & Antifungal & A. brassicae, A. niger & [108] \\
\hline Penicitide A & Antifungal & A. brassicae, A. niger & [107] \\
\hline Penicyclones A-E & Antibacterial & S. aureus & [91] \\
\hline Perinadine A & Antibacterial & B. subtilis, M. luteus & [59] \\
\hline Pinodiketopiperazine A & Antibacterial & E. coli & [132] \\
\hline \multirow{2}{*}{ Scalusamide A } & Antibacterial & M. luteus & \multirow{2}{*}{ [60] } \\
\hline & Antifungal & Cryptococcus neoformans & \\
\hline Talaromycesones & Antibacterial & S. aureus m.r., S. epidermidis & [161] \\
\hline Terretrione D & Antifungal & C. albicans & [167] \\
\hline Xestodecalactone B & Antifungal & C. albicans & [47] \\
\hline
\end{tabular}

m.r.: Methicillin resistant. 
A few compounds displayed consistent effects against important viruses, such as the influenza virus A (H1N1) and HIV-1. Particularly, the cytopathic effects induced by the former in MDCK cells were found to be inhibited by sorbicathecols A-B (Figure 7) [153], while the latter was impaired by brevione F, which inhibited its replication in C8166 cells [92], and sorbicillactone A, which inhibited the expression of viral proteins and protected $\mathrm{H} 9$ cells (human $\mathrm{T}$ lymphocytes) against cytopathic effects [62].

Finally, some miscellaneous bioactive effects can be mentioned for a few compounds (Figure 8).

In the search for novel products to be used as additives in antifouling paints used as protective coats for ships' hulls, potent activities against the larval settlement of barnacles (Balanus amphitrite) were evidenced for 6,8,5' $6^{\prime}$-tetrahydroxy-3'-methylflavone [136], and talaromycin C [162].

Widely considered as a model organism used to test the toxicity of chemicals, brine shrimp (Artemia salina) has been employed for demonstrating the toxic effects of products such as 6,7-dihydroxy-3-methoxy-3-methylphthalide [132], 13-O-prenyl-26-hydroxyverruculogen [148], adametizine A [155], the peniciadametizines [156], and the communesins [51]. The latter represent a numerically expanding series of cytochalasan alkaloids which have been also introduced to some extent for cytotoxic/antiproliferative properties (Table 2), and insecticidal effects resulting after oral administration to silkworms [215]. Insect neurotoxicity was also observed in assays carried out on larvae of the bluebottle fly (Calliphora vomitoria) [94]. Moreover, in a study employing a zebra-fish model, communesin I and two more novel compounds, fumiquinazoline $\mathrm{Q}$ and protuboxepin $\mathrm{E}$, were reported for cardiotonic effects, as well as some extent of vasculogenetic properties assessed with reference to both number and length of vessels [168].

\section{Conclusions}

Data summarized in this review highlight the widespread occurrence at sea of Penicillium/Talaromyces strains, and their extraordinary potential as a source of novel bioactive compounds and drugs. As new data accumulate more and more, the awareness is increasing within the scientific community that these microorganisms represent a trove of unexplored biodiversity, and that more exhaustive investigations should be carried out. In this regard, a comprehensive work was recently published concerning diversity and antifungal properties of a group of 184 marine strains belonging to 36 different Penicillium species from Korea [216]. Also a comparison shows that as many as 18 of these species were not even mentioned in our review, which makes it very likely that our proposed record series will be considerably expanded if this biological material is further characterized in order to detect the biochemical determinants of the fungitoxic effects.

In the meantime, much work is to be done with reference to more complete characterization of the biological activities of the material accumulated so far, in view of the increasing number of products which can evolve to the drug level. A good example is represented by sorbicillactone A, whose notable antileukemic effects have stimulated studies for improving its laboratory yields in view of large scale production [217]. However, progress towards this ultimate step is largely dependent on the extent to which the pharmaceutical industry will prove to be prepared to grasp such a great opportunity. It is desirable that the recent policy adopted by most governments worldwide aimed at involving the manufacturing industry in funding for basic research, turns into a more decisive effort, with ensuing results, in this direction.

Acknowledgments: Antonio Trincone acknowledges BENTEN project within the Biotechnology Network of Campania Region (Italy).

Conflicts of Interest: The authors declare no conflict of interest.

\section{References}

1. Jones, E.B.G.; Pang, K.L. Introduction marine fungi. In Marine Fungi and Fungal-Like Organisms; Walter de Gruyter: Berlin, Germany, 2012; pp. 1-13. 
2. Kohlmeyer, J.; Kohlmeyer, E. Marine Mycology: The Higher Fungi; Elsevier: Philadelphia, PA, USA, $2013 ;$ p. 704.

3. König, G.M.; Kehraus, S.; Seibert, S.F.; Abdel-Lateff, A.; Müller, D. Natural products from marine organisms and their associated microbes. ChemBioChem 2006, 7, 229-238. [CrossRef] [PubMed]

4. Sallenave-Namont, C.; Pouchus, Y.F.; Du Pont, T.R.; Lassus, P.; Verbist, J.F. Toxigenic saprophytic fungi in marine shellfish farming areas. Mycopathologia 2000, 149, 21-25. [CrossRef] [PubMed]

5. Marrouchi, R.; Benoit, E.; Le Caer, J.P.; Belayouni, N.; Belghith, H.; Molgó, J.; Kharrat, R. Toxic C17-sphinganine analogue mycotoxin, contaminating Tunisian mussels, causes flaccid paralysis in rodents. Mar. Drugs 2013, 11, 4724-4740. [CrossRef] [PubMed]

6. Rateb, M.E.; Ebel, R. Secondary metabolites of fungi from marine habitats. Nat. Prod. Rep. 2011, 28, $290-344$. [CrossRef] [PubMed]

7. Baker, D.D.; Chu, M.; Oza, U.; Rajgarhia, V. The value of natural products to future pharmaceutical discovery. Nat. Prod. Rep. 2007, 24, 1225-1244. [CrossRef] [PubMed]

8. Dias, D.A.; Urban, S.; Roessner, U. A historical overview of natural products in drug discovery. Metabolites 2012, 2, 303-336. [CrossRef] [PubMed]

9. Lin, X.; Zhou, X.; Wang, F.; Liu, K.; Yang, B.; Yang, X.; Peng, Y.; Liu, J.; Ren, Z.; Liu, Y. A new cytotoxic sesquiterpene quinone produced by Penicillium sp. F00120 isolated from a deep sea sediment sample. Mar. Drugs 2012, 10, 106-115. [CrossRef] [PubMed]

10. Qi, J.; Shao, C.L.; Liu, M.; Qi, X.; Wang, C.Y. Bioactive steroids from a marine-derived fungus Penicillium sp. from the South China Sea. Chem. Nat. Comp. 2014, 50, 568-570. [CrossRef]

11. Stocker-Wörgötter, E. Metabolic diversity of lichen-forming ascomycetous fungi: Culturing, polyketide and shikimate metabolite production, and PKS genes. Nat. Prod. Rep. 2008, 25, 188-200. [CrossRef] [PubMed]

12. Gao, S.S.; Li, X.M.; Zhang, Y.; Li, C.S.; Cui, C.M.; Wang, B.G. Comazaphilones A-F, azaphilone derivatives from the marine sediment-derived fungus Penicillium commune QSD-17. J. Nat. Prod. 2011, 74, 256-261. [CrossRef] [PubMed]

13. Chai, Y.J.; Cui, C.B.; Li, C.W.; Wu, C.J.; Tian, C.K.; Hua, W. Activation of the dormant secondary metabolite production by introducing gentamicin-resistance in a marine-derived Penicillium purpurogenum G59. Mar. Drugs 2012, 10, 559-582. [CrossRef] [PubMed]

14. Fang, S.M.; Cui, C.B.; Li, C.W.; Wu, C.J.; Zhang, Z.J.; Li, L.; Haung, X.J.; Ye, W.C. Purpurogemutantin and purpurogemutantidin, new drimenyl cyclohexenone derivatives produced by a mutant obtained by diethyl sulfate mutagenesis of a marine-derived Penicillium purpurogenum G59. Mar. Drugs 2012, 10, 1266-1287. [CrossRef] [PubMed]

15. Wu, C.-J.; Li, C.-W.; Cui, C.-B. Seven new and two known lipopeptides as well as five known polyketides: The activated production of silent metabolites in a marine-derived fungus by chemical mutagenesis strategy using diethyl sulphate. Mar. Drugs 2014, 12, 1815-1838. [CrossRef] [PubMed]

16. Chen, H.; Aktas, N.; Konuklugil, B.; Mándi, A.; Daletos, G.; Lin, W.; Dai, H.; Kurtan, T.; Proksch, P. A new fusarielin analogue from Penicillium sp. isolated from the Mediterranean sponge Ircinia oros. Tetrahedron Lett. 2015, 56, 5317-5320. [CrossRef]

17. Taylor, J.W. One fungus = one name: DNA and fungal nomenclature twenty years after PCR. IMA Fungus 2011, 2, 113-120. [CrossRef] [PubMed]

18. Samson, R.A.; Yilmaz, N.; Houbraken, J.; Spierenburg, H.; Seifert, K.A.; Peterson, S.W.; Varga, J.; Frisvad, J.C. Phylogeny and nomenclature of the genus Talaromyces and taxa accommodated in Penicillium subgenus Biverticillium. Stud. Mycol. 2011, 70, 159-183. [CrossRef] [PubMed]

19. Houbraken, J.; Samson, R.A. Phylogeny of Penicillium and the segregation of Trichocomaceae into three families. Stud. Mycol. 2011, 70,1-51. [CrossRef]

20. Yilmaz, N.; Visagie, C.M.; Houbraken, J.; Frisvad, J.C.; Samson, R.A. Polyphasic taxonomy of the genus Talaromyces. Stud. Mycol. 2014, 78, 175-341. [CrossRef] [PubMed]

21. Frisvad, J.C.; Andersen, B.; Thrane, U. The use of secondary metabolite profiling in chemotaxonomy of filamentous fungi. Mycol. Res. 2008, 112, 231-240. [CrossRef] [PubMed]

22. Demain, A.L.; Elander, R.P. The $\beta$-lactam antibiotics: Past, present, and future. Antonie Van Leeuwenhoek 1999, 75, 5-19. [CrossRef] [PubMed]

23. Chakravarti, R.; Sahai, V. Compactin-A review. Appl. Microbiol. Biotechnol. 2004, 64, 618-624. [CrossRef] [PubMed] 
24. Nicoletti, R.; Ciavatta, M.L.; Buommino, E.; Tufano, M.A. Antitumor extrolites produced by Penicillium species. Int. J. Biomed. Pharm. Sci. 2008, 2, 1-23.

25. Frisvad, J.C.; Smedsgaard, J.; Larsen, T.O.; Samson, R.A. Mycotoxins, drugs and other extrolites produced by species in Penicillium subgenus Penicillium. Stud. Mycol. 2004, 49, 201-241.

26. Jones, E.G.; Suetrong, S.; Sakayaroj, J.; Bahkali, A.H.; Abdel-Wahab, M.A.; Boekhout, T.; Pang, K.L. Classification of marine Ascomycota, Basidiomycota, Blastocladiomycota and Chytridiomycota. Fungal Diver. 2015, 73, 1-72. [CrossRef]

27. Janso, J.E.; Bernan, V.S.; Greenstein, M.; Bugni, T.S.; Ireland, C.M. Penicillium dravuni, a new marine-derived species from an alga in Fiji. Mycologia 2005, 97, 444-453. [CrossRef] [PubMed]

28. Shigemori, H.; Wakuri, S.; Yazawa, K.; Nakamura, T.; Sasaki, T.; Kobayashi, J. Fellutamides A and B, cytotoxic peptides from a marine fish-possessing fungus Penicillium fellutanum. Tetrahedron 1991, 47, 8529-8534. [CrossRef]

29. Numata, A.; Takahashi, C.; Ito, Y.; Takada, T.; Kawai, K.; Usami, Y.; Matsumura, E.; Imachi, M.; Ito, T.; Hasegawa, T. Communesins, cytotoxic metabolites of a fungus isolated from a marine alga. Tetrahedron Lett. 1993, 34, 2355-2358. [CrossRef]

30. Numata, A.; Takahashi, C.; Ito, Y.; Minoura, K.; Yamada, T.; Matsuda, C.; Nomoto, K. Penochalasins, a novel class of cytotoxic cytochalasans from a Penicillium species separated from a marine alga: Structure determination and solution conformation. J. Chem. Soc. Perkin Trans. 1996, 239-245. [CrossRef]

31. Takahashi, C.; Numata, A.; Yamada, T.; Minoura, K.; Enomoto, S.; Konishi, K.; Nakai, M.; Matsuda, C.; Nomoto, K. Penostatins, novel cytotoxic metabolites from a Penicillium species separated from a green alga. Tetrahedron Lett. 1996, 37, 655-658. [CrossRef]

32. Iwamoto, C.; Minoura, K.; Oka, T.; Ohta, T.; Hagishita, S.; Numata, A. Absolute stereostructures of novel cytotoxic metabolites, penostatins A-E, from a Penicillium species separated from an Enteromorpha alga. Tetrahedron 1999, 55, 14353-14368. [CrossRef]

33. Iwamoto, C.; Minoura, K.; Hagishita, S.; Nomoto, K.; Numata, A. Penostatins F-I, novel cytotoxic metabolites from a Penicillium species separated from an Enteromorpha marine alga. J. Chem. Soc. Perkin Trans. 1998, 449-456. [CrossRef]

34. Iwamoto, C.; Yamada, T.; Ito, Y.; Minoura, K.; Numata, A. Cytotoxic cytochalasans from a Penicillium species separated from a marine alga. Tetrahedron 2001, 57, 2997-3004. [CrossRef]

35. Woo, J.T.; Ono, H.; Tsuji, T. Cathestatins, new cysteine protease inhibitors produced by Penicillium citrinum. Biosci. Biotechnol. Biochem. 1995, 59, 350-352. [CrossRef] [PubMed]

36. Kakeya, H.; Takahashi, I.; Okada, G.; Isono, K.; Osada, H. Epolactaene, a novel neuritogenic compound in human neuroblastoma cells, produced by a marine fungus. J. Antibiot. 1995, 48, 733-735. [CrossRef] [PubMed]

37. Cui, C.B.; Usukata, M.; Kakeya, H.; Onose, R.; Okada, G.; Takahashi, I.; Isono, K.; Osada, H. Acetophthalidin, a novel inhibitor of mammalian cell cycle, produced by a fungus isolated from a sea sediment. J. Antibiot. 1996, 49, 216-219. [CrossRef] [PubMed]

38. Cabrera, G.M.; Seldes, A.M. Citrinin derivatives from an intertidal marine Penicillium. An. Asoc. Quim. Argent. 1997, 85, 193-196.

39. Onuki, H.; Miyashige, H.; Hasegawa, H.; Yamashita, S. NI15501A, a novel anthranilamide derivative from a marine fungus Penicillium sp. J. Antibiot. 1998, 51, 442-444. [CrossRef] [PubMed]

40. Amagata, T.; Minoura, K.; Numata, A. Cytotoxic metabolites produced by a fungal strain from a Sargassum alga. J. Antibiot. 1998, 51, 432-434. [CrossRef]

41. Christophersen, C.; Crescente, O.; Frisvad, J.C.; Gram, L.; Nielsen, J.; Nielsen, P.H.; Rahbæk, L. Antibacterial activity of marine-derived fungi. Mycopathologia 1998, 143, 135-138. [CrossRef] [PubMed]

42. Malmstrøm, J.; Christophersen, C.; Frisvad, J.C. Secondary metabolites characteristic of Penicillium citrinum, Penicillium steckii and related species. Phytochemistry 2000, 54, 301-309. [CrossRef]

43. Son, B.W.; Jensen, P.R.; Kauffman, C.A.; Fenical, W. New cytotoxic epidithiodioxopiperazines related to verticillin A from a marine isolate of the fungus Penicillium. Nat. Prod. Lett. 1999, 13, 213-222. [CrossRef]

44. Kagata, T.; Shigemori, H.; Mikami, Y.; Kobayashi, J. Coruscol A, a new metabolite from the marine-derived fungus Penicillium species. J. Nat. Prod. 2000, 63, 886-887. [CrossRef] 
45. Komatsu, K.; Shigemori, H.; Mikami, Y.; Kobayashi, J. Sculezonones A and B, two metabolites possessing a phenalenone skeleton from a marine-derived fungus Penicillium species. J. Nat. Prod. 2000, 63, 408-409. [CrossRef] [PubMed]

46. Lin, Y.; Shao, Z.; Jiang, G.; Zhou, S.; Cai, J.; Vrijmoed, L.L.P.; Jones, E.G. Penicillazine, a unique quinolone derivative with $4 \mathrm{H}-5$, 6-dihydro-1, 2-oxazine ring system from the marine fungus Penicillium sp. (strain\# 386) from the South China Sea. Tetrahedron 2000, 56, 9607-9609.

47. Edrada, R.A.; Heubes, M.; Brauers, G.; Wray, V.; Berg, A.; Gräfe, U.; Wohlfarth, M.; Mühlbacher, J.; Schaumann, K.; Sudarsono, S.; et al. Online analysis of xestodecalactones A-C, novel bioactive metabolites from the fungus Penicillium cf. montanense and their subsequent isolation from the sponge Xestospongia exigua. J. Nat. Prod. 2002, 65, 1598-1604. [PubMed]

48. Amagata, T.; Amagata, A.; Tenney, K.; Valeriote, F.A.; Lobkovsky, E.; Clardy, J.; Crews, P. Unusual C25 steroids produced by a sponge-derived Penicillium citrinum. Org. Lett. 2003, 5, 4393-4396. [CrossRef] [PubMed]

49. Bugni, T.S.; Bernan, V.S.; Greenstein, M.; Janso, J.E.; Maiese, W.M.; Mayne, C.L.; Ireland, C.M. Brocaenols A-C: Novel polyketides from a marine-derived Penicillium brocae. J. Org. Chem. 2003, 68, 2014-2017. [CrossRef] [PubMed]

50. Bugni, T.S.; Janso, J.E.; Williamson, R.T.; Feng, X.; Bernan, V.S.; Greenstein, M.; Carter, G.T.; Maiese, W.M.; Ireland, C.M. Dictyosphaeric acids A and B: New decalactones from an undescribed Penicillium sp. obtained from the alga Dictyosphaeria versluyii. J. Nat. Prod. 2004, 67, 1396-1399. [CrossRef] [PubMed]

51. Jadulco, R.; Edrada, R.A.; Ebel, R.; Berg, A.; Schaumann, K.; Wray, V.; Steube, K.; Proksch, P. New communesin derivatives from the fungus Penicillium sp. derived from the Mediterranean sponge Axinella verrucosa. J. Nat. Prod. 2004, 67, 78-81. [CrossRef] [PubMed]

52. Bringmann, G.; Lang, G.; Steffens, S.; Schaumann, K. Petrosifungins A and B, novel cyclodepsipeptides from a sponge-derived strain of Penicillium brevicompactum. J. Nat. Prod. 2004, 67, 311-315. [CrossRef] [PubMed]

53. Gautschi, J.T.; Amagata, T.; Amagata, A.; Valeriote, F.A.; Mooberry, S.L.; Crews, P. Expanding the strategies in natural product studies of marine-derived fungi: A chemical investigation of Penicillium obtained from deep water sediment. J. Nat. Prod. 2004, 67, 362-367. [CrossRef] [PubMed]

54. Vansteelandt, M.; Kerzaon, I.; Blanchet, E.; Tankoua, O.F.; Du Pont, T.R.; Joubert, Y.; Monteau, F.; Le Bizec, B.; Frisvad, J.C.; Pouchus, Y.F.; et al. Patulin and secondary metabolite production by marine-derived Penicillium strains. Fungal Biol. 2012, 116, 954-961. [CrossRef] [PubMed]

55. Petit, K.E.; Mondeguer, F.; Roquebert, M.F.; Biard, J.F.; Pouchus, Y.F. Detection of griseofulvin in a marine strain of Penicillium waksmanii by ion trap mass spectrometry. J. Microbiol. Meth. 2004, 58, 59-65. [CrossRef]

56. Vansteelandt, M.; Blanchet, E.; Egorov, M.; Petit, F.; Toupet, L.; Bondon, A.; Monteau, F.; Le Bizec, B.; Thomas, O.; Pouchus, Y.F.; et al. Ligerin, an antiproliferative chlorinated sesquiterpenoid from a marine-derived Penicillium strain. J. Nat. Prod. 2013, 76, 297-301. [CrossRef] [PubMed]

57. Tsuda, M.; Kasai, Y.; Komatsu, K.; Sone, T.; Tanaka, M.; Mikami, Y.; Kobayashi, J. Citrinadin A, a novel pentacyclic alkaloid from marine-derived fungus Penicillium citrinum. Org. Lett. 2004, 6, 3087-3089. [CrossRef] [PubMed]

58. Mugishima, T.; Tsuda, M.; Kasai, Y.; Ishiyama, H.; Fukushi, E.; Kawabata, J.; Watanabe, M.; Akao, K.; Kobayashi, J. Absolute stereochemistry of citrinadins A and B from marine-derived fungus. J. Org. Chem. 2005, 70, 9430-9435. [CrossRef] [PubMed]

59. Sasaki, M.; Tsuda, M.; Sekiguchi, M.; Mikami, Y.; Kobayashi, J. Perinadine A, a novel tetracyclic alkaloid from marine-derived fungus Penicillium citrinum. Org. Lett. 2005, 7, 4261-4264. [CrossRef] [PubMed]

60. Tsuda, M.; Sasaki, M.; Mugishima, T.; Komatsu, K.; Sone, T.; Tanaka, M.; Mikami, Y.; Kobayashi, J. Scalusamides AC, new pyrrolidine alkaloids from the marine-derived fungus Penicillium citrinum. J. Nat. Prod. 2005, 68, 273-276. [CrossRef] [PubMed]

61. He, J.; Lion, U.; Sattler, I.; Gollmick, F.A.; Grabley, S.; Cai, J.; Meiners, M.; Schünke, H.; Schaumann, K.; Dechert, U.; et al. Diastereomeric quinolinone alkaloids from the marine-derived fungus Penicillium janczewskii. J. Nat. Prod. 2005, 68, 1397-1399. [CrossRef]

62. Bringmann, G.; Lang, G.; Gulder, T.A.M.; Hideyuki, H.; Mühlbacher, J.; Maksimenka, K.; Steffens, S.; Schaumann, K.; Stöhr, R.; Wiese, J.; et al. The first sorbicillinoid alkaloids, the antileukemic sorbicillactones A and B, from a sponge derived Penicillium chrysogenum strain. Tetrahedron 2005, 61, 7252-7265. [CrossRef] 
63. Bringmann, G.; Lang, G.; Bruhn, T.; Schäffler, K.; Steffens, S.; Schmaljohann, R.; Wiese, J.; Imhoff, J.F. Sorbifuranones A-C, sorbicillinoid metabolites from Penicillium strains isolated from Mediterranean sponges. Tetrahedron 2010, 66, 9894-9901. [CrossRef]

64. Chen, L.; Fang, Y.; Zhu, T.; Gu, Q.; Zhu, W. Gentisyl alcohol derivatives from the marine-derived fungus Penicillium terrestre. J. Nat. Prod. 2008, 71, 66-70. [CrossRef] [PubMed]

65. Chen, L.; Zhu, T.; Ding, Y.; Khan, I.A.; Gu, Q.; Li, D. Sorbiterrin A, a novel sorbicillin derivative with cholinesterase inhibition activity from the marine-derived fungus Penicillium terrestre. Tetrahedron Lett. 2012, 53, 325-328. [CrossRef]

66. Liu, W.; Gu, Q.; Zhu, W.; Cui, C.; Fan, G. Dihydrotrichodimerol and tetrahydrotrichodimerol, two new bisorbicillinoids, from a marine-derived Penicillium terrestre. J. Antibiot. 2005, 58, 621-624. [CrossRef]

67. Liu, W.; Gu, Q.; Zhu, W.; Cui, C.; Fan, G. Two new benzoquinone derivatives and two new bisorbicillinoids were isolated from a marine-derived fungus Penicillium terrestre. J. Antibiot. 2005, 58, 441-446. [CrossRef] [PubMed]

68. Liu, W.; Gu, Q.; Zhu, W.; Cui, C.; Fan, G.; Zhu, T.; Liu, H.; Fang, Y. Penicillones A and B, two novel polyketides with tricyclo [5.3.1.0.3.8 $]$ undecane skeleton, from a marine-derived fungus Penicillium terrestre. Tetrahedron Lett. 2005, 46, 4993-4996. [CrossRef]

69. Li, D.; Chen, L.; Zhu, T.; Kurtán, T.; Mándi, A.; Zhao, Z.; Li, J.; Gu, Q. Chloctanspirones A and B, novel chlorinated polyketides with an unprecedented skeleton, from marine sediment derived fungus Penicillium terrestre. Tetrahedron 2011, 67, 7913-7918. [CrossRef]

70. Xue, C.; Li, T.; Deng, Z.; Fu, H.; Lin, W. Janthinolide A-B, two new 2,5-piperazinedione derivatives from the endophytic Penicillium janthinellum isolated from the soft coral Dendronephthya sp. Pharmazie 2006, 61, 1041-1044. [CrossRef]

71. Rovirosa, J.; Diaz-Marrero, A.N.A.; Darías, J.; Painemal, K.; San Martín, A. Secondary metabolites from marine Penicillium brevicompactum. J. Chil. Chem. Soc. 2006, 51, 775-778. [CrossRef]

72. Lang, G.; Wiese, J.; Schmaljohann, R.; Imhoff, J.F. New pentaenes from the sponge-derived marine fungus Penicillium rugulosum: Structure determination and biosynthetic studies. Tetrahedron 2007, 63, 11844-11849. [CrossRef]

73. El-Beih, A.A.; Kato, H.; Tsukamoto, S.; Ohta, T. CYP3A4 inhibitors isolated from a marine derived fungus Penicillium species. J. Nat. Med. 2007, 61, 175-177. [CrossRef]

74. Smetanina, O.F.; Kalinovsky, A.I.; Khudyakova, Y.V.; Pivkin, M.V.; Dmitrenok, P.S.; Fedorov, S.N.; Ji, H.; Kwak, J.-Y.; Kuznetsova, T.A. Indole alkaloids produced by a marine fungus isolate of Penicillium janthinellum Biourge. J. Nat. Prod. 2007, 70, 906-909. [CrossRef] [PubMed]

75. Capon, R.J.; Stewart, M.; Ratnayake, R.; Lacey, E.; Gill, J.H. Citromycetins and bilains A-C: New aromatic polyketides and diketopiperazines from Australian marine-derived and terrestrial Penicillium spp. J. Nat. Prod. 2007, 70, 1746-1752. [CrossRef] [PubMed]

76. Zhang, D.; Li, X.; Kang, J.S.; Choi, H.D.; Jung, J.H.; Son, B.W. Redoxcitrinin, a biogenetic precursor of citrinin from marine isolate of fungus Penicillium sp. J. Microbiol. Biotechnol. 2007, 17, 865-867. [PubMed]

77. Xin, Z.; Fang, Y.; Du, L.; Zhu, T.; Duan, L.; Chen, J.; Gu, Q.; Zhu, W. Aurantiomides A-C, quinazoline alkaloids from the sponge-derived fungus Penicillium aurantiogriseum SP0-19. J. Nat. Prod. 2007, 70, 853-855. [CrossRef] [PubMed]

78. Xin, Z.H.; Zhu, T.J.; Wang, W.L.; Du, L.; Fang, Y.C.; Gu, Q.Q.; Zhu, W.M. Isocoumarin derivatives from the sea squirt-derived fungus Penicillium stoloniferum QY2-10 and the halotolerant fungus Penicillium notatum B-52. Arch. Pharm. Res. 2007, 30, 816-819. [CrossRef] [PubMed]

79. Ren, H.; Gu, Q.Q.; Cui, C.B. Anthraquinone derivatives produced by marine-derived Penicillium flavidorsum SHK1-27 and their antitumor activities. Chin. J. Med. Chem. 2007, 17, 148-154.

80. Iida, M.; Ooi, T.; Kito, K.; Yoshida, S.; Kanoh, K.; Shizuri, Y.; Kusumi, T. Three new polyketide-terpenoid hybrids from Penicillium sp. Org. Lett. 2008, 10, 845-848. [CrossRef] [PubMed]

81. Huang, Y.F.; Qiao, L.; Lv, A.L.; Pei, Y.H.; Tian, L. Eremophilane sesquiterenes from the marine fungus Penicillium sp. BL27-2. Chin. Chem. Lett. 2008, 19, 562-564. [CrossRef]

82. Motohashi, K.; Hashimoto, J.; Inaba, S.; Khan, S.T.; Komaki, H.; Nagai, A.; Takagi, M.; Shin-ya, K. New sesquiterpenes, JBIR-27 and-28, isolated from a tunicate-derived fungus, Penicillium sp. SS080624SCf1. J. Antibiot. 2009, 62, 247-250. [CrossRef] [PubMed] 
83. Mizushina, Y.; Motoshima, H.; Yamaguchi, Y.; Takeuchi, T.; Hirano, K.; Sugawara, F.; Yoshida, H. 3-O-methylfunicone, a selective inhibitor of mammalian Y-family DNA polymerases from an Australian sea salt fungal strain. Mar. Drugs 2009, 7, 624-639. [CrossRef] [PubMed]

84. De Silva, E.D.; Geiermann, A.S.; Mitova, M.I.; Kuegler, P.; Blunt, J.W.; Cole, A.L.; Munro, M.H. Isolation of 2-pyridone alkaloids from a New Zealand marine-derived Penicillium species. J. Nat. Prod. 2009, 72, 477-479. [CrossRef] [PubMed]

85. Zhu, T.J.; Du, L.; Hao, P.F.; Lin, Z.J.; Gu, Q.Q. Citrinal A, a novel tricyclic derivative of citrinin, from an algicolous fungus Penicillium sp. i-1-1. Chin. Chem. Lett. 2009, 20, 917-920. [CrossRef]

86. Gamal-Eldeen, A.M.; Abdel-Lateff, A.; Okino, T. Modulation of carcinogen metabolizing enzymes by chromanone A; a new chromone derivative from algicolous marine fungus Penicillium sp. Environ. Toxicol. Pharmacol. 2009, 28, 317-322. [CrossRef] [PubMed]

87. Du, L.; Yang, X.; Zhu, T.; Wang, F.; Xiao, X.; Park, H.; Gu, Q. Diketopiperazine alkaloids from a deep ocean sediment derived fungus Penicillium sp. Chem. Pharm. Bull. 2009, 57, 873-876. [CrossRef] [PubMed]

88. Du, L.; Li, D.; Zhu, T.; Cai, S.; Wang, F.; Xiao, X.; Gu, Q. New alkaloids and diterpenes from a deep ocean sediment derived fungus Penicillium sp. Tetrahedron 2009, 65, 1033-1039. [CrossRef]

89. Du, L.; Feng, T.; Zhao, B.; Li, D.; Cai, S.; Zhu, T.; Wang, F.; Xiao, X.; Gu, Q. Alkaloids from a deep ocean sediment-derived fungus Penicillium sp. and their antitumor activities. J. Antibiot. 2010, 63, 165-170. [CrossRef] [PubMed]

90. Guo, W.; Peng, J.; Zhu, T.; Gu, Q.; Keyzers, R.A.; Li, D. Sorbicillamines A-E, nitrogen-containing sorbicillinoids from the deep-sea-derived fungus Penicillium sp. F23-2. J. Nat. Prod. 2013, 76, 2106-2112. [CrossRef] [PubMed]

91. Guo, W.; Zhang, Z.; Zhu, T.; Gu, Q.; Li, D. Penicyclones A-E, antibacterial polyketides from the deep-sea-derived fungus Penicillium sp. F23-2. J. Nat. Prod. 2015, 78, 2699-2703. [CrossRef] [PubMed]

92. Li, Y.; Ye, D.; Chen, X.; Lu, X.; Shao, Z.; Zhang, H.; Che, Y. Breviane spiroditerpenoids from an extreme-tolerant Penicillium sp. isolated from a deep sea sediment sample. J. Nat. Prod. 2009, 72, 912-916. [CrossRef] [PubMed]

93. Li, Y.; Ye, D.; Shao, Z.; Cui, C.; Che, Y. A sterol and spiroditerpenoids from a Penicillium sp. isolated from a deep sea sediment sample. Mar. Drugs 2012, 10, 497-508. [CrossRef] [PubMed]

94. Kerzaon, I.; Pouchus, Y.F.; Monteau, F.; Le Bizec, B.; Nourrisson, M.R.; Biard, J.F.; Grovel, O. Structural investigation and elucidation of new communesins from a marine-derived Penicillium expansum Link by liquid chromatography/electrospray ionization mass spectrometry. Rapid Commun. Mass Spectrom. 2009, 23, 3928-3938. [CrossRef]

95. Trisuwan, K.; Rukachaisirikul, V.; Sukpondma, Y.; Phongpaichit, S.; Preedanon, S.; Sakayaroj, J. Lactone derivatives from the marine-derived fungus Penicillium sp. PSU-F44. Chem. Pharm. Bull. 2009, 57, 1100-1102. [CrossRef] [PubMed]

96. Trisuwan, K.; Rukachaisirikul, V.; Sukpondma, Y.; Phongpaichit, S.; Preedanon, S.; Sakayaroj, J. Furo[3,2-h]isochroman, furo[3,2-h]isoquinoline, isochroman, phenol, pyranone, and pyrone derivatives from the sea fan-derived fungus Penicillium sp. PSU-F40. Tetrahedron 2010, 66, 4484-4489. [CrossRef]

97. Liu, S.; Yan, X.; Yu, M.; Chen, J.; Zhang, L. A novel compound from Penicillium sp. (M207142). Chem. Nat. Comp. 2010, 46, 116-118. [CrossRef]

98. Wiese, J.; Ohlendorf, B.; Blümel, M.; Schmaljohann, R.; Imhoff, J.F. Phylogenetic identification of fungi isolated from the marine sponge Tethya aurantium and identification of their secondary metabolites. Mar. Drugs 2011, 9, 561-585. [CrossRef]

99. Ueda, J.Y.; Hashimoto, J.; Inaba, S.; Takagi, M.; Shin-ya, K. JBIR-59, a new sorbicillinoid, from a marine-derived fungus Penicillium citrinum SpI080624G1f01. J. Antibiot. 2010, 63, 203-205. [CrossRef] [PubMed]

100. Kawahara, T.; Takagi, M.; Shin-ya, K. JBIR-124: A novel antioxidative agent from a marine sponge-derived fungus Penicillium citrinum SpI080624G1f01. J. Antibiot. 2012, 65, 45-47. [CrossRef] [PubMed]

101. Pimenta, E.F.; Vita-Marques, A.M.; Tininis, A.; Seleghim, M.H.R.; Sette, L.D.; Veloso, K.; Ferreira, A.G.; Williams, D.E.; Patrick, B.O.; Dalisay, D.S.; et al. Use of experimental design for the optimization of the production of new secondary metabolites by two Penicillium species. J. Nat. Prod. 2010, 73, 1821-1832. [CrossRef] [PubMed] 
102. Zhou, L.N.; Zhu, T.J.; Cai, S.X.; Gu, Q.Q.; Li, D.H. Three new indole-containing diketopiperazine alkaloids from a deep-ocean sediment derived fungus Penicillium griseofulvum. Helv. Chim. Acta 2010, 93, 1758-1763. [CrossRef]

103. Yu, K.; Ren, B.; Wei, J.; Chen, C.; Sun, J.; Song, F.; Dai, H.; Zhang, L. Verrucisidinol and verrucosidinol acetate, two pyrone-type polyketides isolated from a marine derived fungus, Penicillium aurantiogriseum. Mar. Drugs 2010, 8, 2744-2754. [CrossRef] [PubMed]

104. Song, F.; Ren, B.; Yu, K.; Chen, C.; Guo, H.; Yang, N.; Gao, H.; Liu, X.; Liu, M.; Tong, Y.; et al. Quinazolin-4-one coupled with pyrrolidin-2-iminium alkaloids from marine-derived fungus Penicillium aurantiogriseum. Mar. Drugs 2012, 10, 1297-1306. [CrossRef] [PubMed]

105. Yang, G.; Yun, K.; Nenkep, V.N.; Choi, H.D.; Kang, J.S.; Son, B.W. Induced production of halogenated diphenyl ethers from the marine-derived fungus Penicillium chrysogenum. Chem. Biodivers. 2010, 7, 2766-2770. [CrossRef] [PubMed]

106. Meyer, S.W.; Mordhorst, T.F.; Lee, C.; Jensen, P.R.; Fenical, W.; Köck, M. Penilumamide, a novel lumazine peptide isolated from the marine-derived fungus, Penicillium sp. CNL-338. Org. Biomol. Chem. 2010, 8, 2158-2163. [CrossRef] [PubMed]

107. Gao, S.S.; Li, X.M.; Du, F.Y.; Li, C.S.; Proksch, P.; Wang, B.G. Secondary metabolites from a marine-derived endophytic fungus Penicillium chrysogenum QEN-24S. Mar. Drugs 2011, 9, 59-70. [CrossRef] [PubMed]

108. Gao, S.S.; Li, X.M.; Li, C.S.; Proksch, P.; Wang, B.G. Penicisteroids A and B, antifungal and cytotoxic polyoxygenated steroids from the marine alga-derived endophytic fungus Penicillium chrysogenum QEN-24S. Bioorg. Med. Chem. Lett. 2011, 21, 2894-2897. [CrossRef] [PubMed]

109. Gao, S.S.; Li, X.M.; Zhang, Y.; Li, C.S.; Wang, B.G. Conidiogenones H and I, two new diterpenes of cyclopiane class from a marine-derived endophytic fungus Penicillium chrysogenum QEN-24S. Chem. Biodivers. 2011, 8, 1748-1753. [CrossRef] [PubMed]

110. Smetanina, O.F.; Yurchenko, A.N.; Kalinovsky, A.I.; Pushilin, M.A.; Slinkina, N.N.; Yurchenko, E.A.; Afiyatullov, S.S. 4-Methoxy-3-methylgoniothalamin from marine-derived fungi of the genus Penicillium. Russ. Chem. Bull. 2011, 60, 760-763. [CrossRef]

111. Liu, Q.Y.; Zhou, T.; Zhao, Y.Y.; Chen, L.; Gong, M.W.; Xia, Q.W.; Ying, M.G.; Zheng, Q.H.; Zhang, Q.Q. Antitumor effects and related mechanisms of penicitrinine $\mathrm{A}$, a novel alkaloid with a unique spiro skeleton from the marine fungus Penicillium citrinum. Mar. Drugs 2015, 13, 4733-4753. [CrossRef] [PubMed]

112. Chen, L.; Liu, W.; Hu, X.; Huang, K.; Wu, J.L.; Zhang, Q.Q. Citrinin derivatives from the marine-derived fungus Penicillium citrinum. Chem. Pharm. Bull. 2011, 59, 515-517. [CrossRef] [PubMed]

113. Chen, L.; Gong, M.-W.; Peng, Z.-F.; Zhou, T.; Ying, M.-G.; Zheng, Q.-H.; Liu, Q.-Y.; Zhang, Q.Q. The marine fungal metabolite, dicitrinone B, induces A375 cell apoptosis through the ROS-related caspase pathway. Mar. Drugs 2014, 12, 1939-1958. [CrossRef] [PubMed]

114. Sun, Y.; Takada, K.; Takemoto, Y.; Yoshida, M.; Nogi, Y.; Okada, S.; Matsunaga, S. Gliotoxin analogues from a marine-derived fungus, Penicillium sp., and their cytotoxic and histone methyltransferase inhibitory activities. J. Nat. Prod. 2011, 75, 111-114. [CrossRef] [PubMed]

115. Li, J.L.; Zhang, P.; Lee, Y.M.; Hong, J.; Yoo, E.S.; Bae, K.S.; Jung, J.H. Oxygenated hexylitaconates from a marine sponge-derived fungus Penicillium sp. Chem. Pharm. Bull. 2011, 59, 120-123. [CrossRef] [PubMed]

116. Li, C.S.; An, C.Y.; Li, X.M.; Gao, S.S.; Cui, C.M.; Sun, H.F.; Wang, B.G. Triazole and dihydroimidazole alkaloids from the marine sediment-derived fungus Penicillium paneum SD-44. J. Nat. Prod. 2011, 74, 1331-1334. [CrossRef]

117. Li, C.S.; Li, X.M.; Gao, S.S.; Lu, Y.H.; Wang, B.G. Cytotoxic anthranilic acid derivatives from deep sea sediment-derived fungus Penicillium paneum SD-44. Mar. Drugs 2013, 11, 3068-3076. [CrossRef] [PubMed]

118. Li, C.; Li, X.; An, C.; Wang, B. Prenylated indole alkaloid derivatives from marine sediment-derived fungus Penicillium paneum SD-44. Helv. Chim. Acta 2014, 97, 1440-1444. [CrossRef]

119. Gao, S.S.; Shang, Z.; Li, X.M.; Li, C.S.; Cui, C.M.; Wang, B.G. Secondary metabolites produced by solid fermentation of the marine-derived fungus Penicillium commune QSD-17. Biosci. Biotechnol. Biochem. 2012, 76, 358-360. [CrossRef] [PubMed]

120. Kossuga, M.H.; Romminger, S.; Xavier, C.; Milanetto, M.C.; do Valle, M.Z.; Pimenta, E.F.; Morais, R.P.; de Carvalho, E.; Mizuno, C.M.; Coradello, L.F.C.; et al. Evaluating methods for the isolation of marine-derived fungal strains and production of bioactive secondary metabolites. Rev. Bras. Farmacogn. 2012, 22, 257-267. [CrossRef] 
121. Gao, H.; Zhang, L.; Zhu, T.; Gu, Q.; Li, D. Unusual pyrrolyl 4-quinolinone alkaloids from the marine-derived fungus Penicillium sp. ghq208. Chem. Pharm. Bull. 2012, 60, 1458-1460. [CrossRef] [PubMed]

122. Myobatake, Y.; Takeuchi, T.; Kuramochi, K.; Kuriyama, I.; Ishido, T.; Hirano, K.; Sugawara, F.; Yoshida, H.; Mizushina, Y. Pinophilins A and B, inhibitors of mammalian A-, B-, and Y-family DNA polymerases and human cancer cell proliferation. J. Nat. Prod. 2012, 75, 135-141. [CrossRef] [PubMed]

123. Kawahara, T.; Takagi, M.; Shin-ya, K. Three new depsipeptides, JBIR-113, JBIR-114 and JBIR-115, isolated from a marine sponge-derived Penicillium sp. fS36. J. Antibiot. 2012, 65, 147-150. [CrossRef] [PubMed]

124. Chen, Z.; Zheng, Z.; Huang, H.; Song, Y.; Zhang, X.; Ma, J.; Wang, B.; Zhang, C.; Ju, J. Penicacids A-C, three new mycophenolic acid derivatives and immunosuppressive activities from the marine-derived fungus Penicillium sp. SOF07. Bioorg. Med. Chem. Lett. 2012, 22, 3332-3335. [CrossRef] [PubMed]

125. Wu, G.; Ma, H.; Zhu, T.; Li, J.; Gu, Q.; Li, D. Penilactones A and B, two novel polyketides from Antarctic deep-sea derived fungus Penicillium crustosum PRB-2. Tetrahedron 2012, 68, 9745-9749. [CrossRef]

126. Shang, Z.; Li, X.; Meng, L.; Li, C.; Gao, S.; Huang, C.; Wang, B. Chemical profile of the secondary metabolites produced by a deepsea sediment-derived fungus Penicillium commune SD-118. Chin. J. Oceanol. Limnol. 2012, 30, 305-314. [CrossRef]

127. Wang, J.; Liu, P.; Wang, Y.; Wang, H.; Li, J.; Zhuang, Y.; Zhu, W. Antimicrobial aromatic polyketides from gorgonian-associated fungus, Penicillium commune 518. Chin. J. Chem. 2012, 30, 1236-1242. [CrossRef]

128. Geiger, M.; Guitton, Y.; Vansteelandt, M.; Kerzaon, I.; Blanchet, E.; Robiou du Pont, T.; Frisvad, J.C.; Hess, P.; Pouchus, Y.F.; Grovel, O. Cytotoxicity and mycotoxin production of shellfish-derived Penicillium spp., a risk for shellfish consumers. Lett. Appl. Microbiol. 2013, 57, 385-392. [CrossRef] [PubMed]

129. Yurchenko, A.N.; Smetanina, O.F.; Kalinovskii, A.I.; Kirichuk, N.N.; Yurchenko, E.A.; Afiyatullov, S.S. Biologically active metabolites of the facultative marine fungus Penicillium citrinum. Chem. Nat. Comp. 2013, 48, 996-998. [CrossRef]

130. Julianti, E.; Lee, J.H.; Liao, L.; Park, W.; Park, S.; Oh, D.C.; Oh, K.B.; Shin, J. New polyaromatic metabolites from a marine-derived fungus Penicillium sp. Org. Lett. 2013, 15, 1286-1289. [CrossRef] [PubMed]

131. Subramani, R.; Kumar, R.; Prasad, P.; Aalbersberg, W. Cytotoxic and antibacterial substances against multi-drug resistant pathogens from marine sponge symbiont: Citrinin, a secondary metabolite of Penicillium sp. Asian Pac. J. Trop. Biomed. 2013, 3, 291-296. [CrossRef]

132. Wang, M.H.; Li, X.M.; Li, C.S.; Ji, N.Y.; Wang, B.G. Secondary metabolites from Penicillium pinophilum SD-272, a marine sediment-derived fungus. Mar. Drugs 2013, 11, 2230-2238. [CrossRef] [PubMed]

133. Flewelling, A.J.; Johnson, J.A.; Gray, C.A. Antimicrobials from the marine algal endophyte Penicillium sp. Nat. Prod. Commun. 2013, 8, 373-374. [PubMed]

134. Qi, J.; Shao, C.L.; Li, Z.Y.; Gan, L.S.; Fu, X.M.; Bian, W.T.; Zhao, H.Y.; Wang, C.Y. Isocoumarin derivatives and benzofurans from a sponge-derived Penicillium sp. fungus. J. Nat. Prod. 2013, 76, 571-579. [CrossRef] [PubMed]

135. He, F.; Han, Z.; Peng, J.; Qian, P.Y.; Qi, S.H. Antifouling indole alkaloids from two marine derived fungi. Nat. Prod. Commun. 2013, 8, 329-332. [PubMed]

136. Bao, J.; Sun, Y.L.; Zhang, X.Y.; Han, Z.; Gao, H.C.; He, F.; Qian, P.Y.; Qi, S.H. Antifouling and antibacterial polyketides from marine gorgonian coral-associated fungus Penicillium sp. SCSGAF 0023. J. Antibiot. 2013, 66, 219-223. [CrossRef] [PubMed]

137. Sohn, J.H.; Lee, D.S.; Oh, H.C. PTP1B inhibitory secondary metabolites from marine-derived fungal strains Penicillium spp. and Eurotium sp. J. Microbiol. Biotechnol. 2013, 23, 1206-1211. [CrossRef] [PubMed]

138. Lee, D.S.; Ko, W.; Quang, T.H.; Kim, K.S.; Sohn, J.H.; Jang, J.H.; Ahn, J.S.; Kim, Y.C.; Oh, H. Penicillinolide A: A new anti-inflammatory metabolite from the marine fungus Penicillium sp. SF-5292. Mar. Drugs 2013, 11, 4510-4526. [CrossRef] [PubMed]

139. Lee, C.; Sohn, J.H.; Jang, J.H.; Ahn, J.S.; Oh, H.; Baltrusaitis, J.; Hwang, I.H.; Gloer, J.B. Cycloexpansamines A and B: Spiroindolinone alkaloids from a marine isolate of Penicillium sp.(SF-5292). J. Antibiot. 2015, 68, 715-718. [CrossRef]

140. Lee, D.S.; Jang, J.H.; Ko, W.; Kim, K.S.; Sohn, J.H.; Kang, M.S.; Ahn, J.S.; Kim, Y.C.; Oh, H. PTP1B inhibitory and anti-inflammatory effects of secondary metabolites isolated from the marine-derived fungus Penicillium sp. JF-55. Mar. Drugs 2013, 11, 1409-1426. [CrossRef] [PubMed]

141. Quang, T.H.; Lee, D.S.; Sohn, J.H.; Kim, Y.C.; Oh, H. A new deoxyisoaustamide derivative from the marine-derived fungus Penicillium sp. JF-72. Bull. Korean Chem. Soc. 2013, 34, 3109-3112. [CrossRef] 
142. An, C.Y.; Li, X.M.; Li, C.S.; Gao, S.S.; Shang, Z.; Wang, B.G. Triazoles and other N-containing metabolites from the marine-derived endophytic fungus Penicillium chrysogenum EN-118. Helv. Chim. Acta 2013, 96, 682-687. [CrossRef]

143. Gao, H.; Zhou, L.; Li, D.; Gu, Q.; Zhu, T.J. New cytotoxic metabolites from the marine-derived fungus Penicillium sp. ZLN29. Helv. Chim. Acta 2013, 96, 514-519. [CrossRef]

144. Scopel, M.; Abraham, W.-R.; Henriques, A.T.; MacEdo, A.J. Dipeptide cis-cyclo(Leucyl-Tyrosyl) produced by sponge associated Penicillium sp. F37 inhibits biofilm formation of the pathogenic Staphylococcus epidermidis. Bioorg. Med. Chem. Lett. 2013, 23, 624-626. [CrossRef] [PubMed]

145. Wu, G.; Lin, A.; Gu, Q.; Zhu, T.; Li, D. Four new chloro-eremophilane sesquiterpenes from an Antarctic deep-sea derived fungus, Penicillium sp. PR19N-1. Mar. Drugs 2013, 11, 1399-1408. [CrossRef] [PubMed]

146. Lin, A.; Wu, G.; Gu, Q.; Zhu, T.; Li, D. New eremophilane-type sesquiterpenes from an Antarctic deep-sea derived fungus, Penicillium sp. PR19 N-1. Arch. Pharm. Res. 2014, 37, 839-844. [CrossRef] [PubMed]

147. Sun, Y.L.; Zhang, X.Y.; Zheng, Z.H.; Xu, X.Y.; Qi, S.H. Three new polyketides from marine-derived fungus Penicillium citrinum SCSGAF 0167. Nat. Prod. Res. 2014, 28, 239-244. [CrossRef] [PubMed]

148. An, C.Y.; Li, X.M.; Li, C.S.; Xu, G.M.; Wang, B.G. Prenylated indolediketopiperazine peroxides and related homologues from the marine sediment-derived fungus Penicillium brefeldianum SD-273. Mar. Drugs 2014, 12, 746-756. [CrossRef] [PubMed]

149. He, J.B.; Ji, Y.N.; Hu, D.B.; Zhang, S.; Yan, H.; Liu, X.C.; Luo, H.R.; Zhu, H.J. Structure and absolute configuration of penicilliumine, a new alkaloid from Penicillium commune 366606. Tetrahedron Lett. 2014, 55, 2684-2686. [CrossRef]

150. Li, X.D.; Miao, F.P.; Liang, X.R.; Ji, N.Y. Meroterpenes from an algicolous strain of Penicillium echinulatum. Magn. Res. Chem. 2014, 52, 247-250. [CrossRef]

151. Liao, L.; Lee, J.H.; You, M.; Choi, T.J.; Park, W.; Lee, S.K.; Oh, D.C.; Oh, K.B.; Shin, J. Penicillipyrones A and B, meroterpenoids from a marine-derived Penicillium sp. fungus. J. Nat. Prod. 2014, 77, 406-410. [CrossRef] [PubMed]

152. Kumla, D.; Dethoup, T.; Buttachon, S.; Singburaudom, N.; Silva, A.M.; Kijjoa, A. Spiculisporic acid E, a new spiculisporic acid derivative and ergosterol derivatives from the marine-sponge associated fungus Talaromyces trachyspermus (KUFA 0021). Nat. Prod. Commun. 2014, 9, 1147-1150.

153. Peng, J.; Zhang, X.; Du, L.; Wang, W.; Zhu, T.; Gu, Q.; Li, D. Sorbicatechols A and B, antiviral sorbicillinoids from the marine-derived fungus Penicillium chrysogenum PJX-17. J. Nat. Prod. 2014, 77, 424-428. [CrossRef] [PubMed]

154. Kim, D.C.; Lee, H.S.; Ko, W.; Lee, D.S.; Sohn, J.H.; Yim, J.H.; Kim, Y.C.; Oh, H. Anti-inflammatory effect of methylpenicinoline from a marine isolate of Penicillium sp. (SF-5995): Inhibition of NF-kB and MAPK pathways in lipopolysaccharide-induced RAW264.7 macrophages and BV2 microglia. Molecules 2014, 19, 18073-18089. [CrossRef] [PubMed]

155. Liu, Y.; Li, X.M.; Meng, L.H.; Jiang, W.L.; Xu, G.M.; Huang, C.G.; Wang, B.G. Bisthiodiketopiperazines and acorane sesquiterpenes produced by the marine-derived fungus Penicillium adametzioides AS-53 on different culture media. J. Nat. Prod. 2015, 78, 1294-1299. [CrossRef] [PubMed]

156. Liu, Y.; Mándi, A.; Li, X.M.; Meng, L.H.; Kurtán, T.; Wang, B.G. Peniciadametizine A, a dithiodiketopiperazine with a unique spiro [furan-2,7'-pyrazino[1,2- $b][1,2]$ oxazine] skeleton, and a related analogue, peniciadametizine B, from the marine sponge-derived fungus Penicillium adametzioides. Mar. Drugs 2015, 13, 3640-3652. [CrossRef] [PubMed]

157. Liu, Y.; Li, X.M.; Meng, L.H.; Wang, B.G. N-Formyllapatin A, a new N-formylspiroquinazoline derivative from the marine-derived fungus Penicillium adametzioides AS-53. Phytochem. Lett. 2014, 10, 145-148. [CrossRef]

158. Quang, T.H.; Ngan, N.T.T.; Ko, W.; Kim, D.C.; Yoon, C.S.; Sohn, J.H.; Yim, J.H.; Kim, Y.C.; Oh, H. Tanzawaic acid derivatives from a marine isolate of Penicillium sp. (SF-6013) with anti-inflammatory and PTP1B inhibitory activities. Bioorg. Med. Chem. Lett. 2014, 24, 5787-5791. [CrossRef] [PubMed]

159. Kildgaard, S.; Mansson, M.; Dosen, I.; Klitgaard, A.; Frisvad, J.C.; Larsen, T.O.; Nielsen, K.F. Accurate dereplication of bioactive secondary metabolites from marine-derived fungi by UHPLC-DAD-QTOFMS and a MS/HRMS library. Mar. Drugs 2014, 12, 3681-3705. [CrossRef] [PubMed] 
160. Zhuravleva, O.I.; Sobolevskaya, M.P.; Afiyatullov, S.S.; Kirichuk, N.N.; Denisenko, V.A.; Dmitrenok, P.S.; Yurchenko, E.A.; Dyshlovoy, S.A. Sargassopenillines A-G, 6,6-spiroketals from the alga-derived fungi Penicillium thomii and Penicillium lividum. Mar. Drugs 2014, 12, 5930-5943. [CrossRef] [PubMed]

161. Wu, B.; Ohlendorf, B.; Oesker, V.; Wiese, J.; Malien, S.; Schmaljohann, R.; Imhoff, J.F. Acetylcholinesterase inhibitors from a marine fungus Talaromyces sp. strain LF458. Mar. Biotechnol. 2015, 17, 110-119. [CrossRef] [PubMed]

162. Chen, M.; Han, L.; Shao, C.L.; She, Z.G.; Wang, C.Y. Bioactive diphenyl ether derivatives from a gorgonian-derived fungus Talaromyces sp. Chem. Biodivers. 2015, 12, 443-450. [CrossRef] [PubMed]

163. Zhao, D.L.; Shao, C.L.; Zhang, Q.; Wang, K.L.; Guan, F.F.; Shi, T.; Wang, C.Y. Azaphilone and diphenyl ether derivatives from a gorgonian-derived strain of the fungus Penicillium pinophilum. J. Nat. Prod. 2015, 78, 2310-2314. [CrossRef] [PubMed]

164. Ngokpol, S.; Suwakulsiri, W.; Sureram, S.; Lirdprapamongkol, K.; Aree, T.; Wiyakrutta, S.; Mahidol, C.; Ruchirawat, S.; Kittakoop, P. Drimane sesquiterpene-conjugated amino acids from a marine isolate of the fungus Talaromyces minioluteus (Penicillium minioluteum). Mar. Drugs 2015, 13, 3567-3580. [CrossRef] [PubMed]

165. Afiyatullov, S.S.; Leshchenko, E.V.; Sobolevskaya, M.P.; Gerasimenko, A.V.; Khudyakova, Y.V.; Kirichuk, N.N.; Mikhailov, V.V. New 3-[2'(R)-hydroxybutyl]-7-hydroxyphthalide from marine isolate of the fungus Penicillium claviforme. Chem. Nat. Comp. 2015, 51, 111-115. [CrossRef]

166. Asiri, I.A.; Badr, J.M.; Youssef, D.T. Penicillivinacine, antimigratory diketopiperazine alkaloid from the marine-derived fungus Penicillium vinaceum. Phytochem. Lett. 2015, 13, 53-58. [CrossRef]

167. Shaala, L.A.; Youssef, D.T. Identification and bioactivity of compounds from the fungus Penicillium sp. CYE-87 isolated from a marine tunicate. Mar. Drugs 2015, 13, 1698-1709. [CrossRef] [PubMed]

168. Fan, Y.-Q.; Li, P.-H.; Chao, Y.-X.; Chen, H.; Du, N.; He, Q.-X.; Liu, K.-C. Alkaloids with cardiovascular effects from the marine-derived fungus Penicillium expansum Y32. Mar. Drugs 2015, 13, 6489-6504. [CrossRef] [PubMed]

169. Kim, J.W.; Ko, S.K.; Son, S.; Shin, K.S.; Ryoo, I.J.; Hong, Y.S.; Oh, H.; Hwang, B.Y.; Hirota, H.; Takahashi, S.; et al. Haenamindole, an unusual diketopiperazine derivative from a marine-derived Penicillium sp. KCB12F005. Bioorg. Med. Chem. Lett. 2015, 25, 5398-5401. [CrossRef] [PubMed]

170. Cardoso-Martínez, F.; de la Rosa, J.M.; Díaz-Marrero, A.R.; Darias, J.; Cerella, C.; Diederich, M.; Cueto, M. Tanzawaic acids isolated from a marine-derived fungus of the genus Penicillium with cytotoxic activities. Org. Biomol. Chem. 2015, 13, 7248-7256. [CrossRef] [PubMed]

171. Bu, Y.Y.; Yamazaki, H.; Takahashi, O.; Kirikoshi, R.; Ukai, K.; Namikoshi, M. Penicyrones A and B, an epimeric pair of $\alpha$-pyrone-type polyketides produced by the marine-derived Penicillium sp. J. Antibiot. 2016, 69, 57-61. [CrossRef]

172. Ding, Z.; Zhang, L.; Fu, J.; Che, Q.; Li, D.; Gu, Q.; Zhu, T. Phenylpyropenes E and F: New meroterpenes from the marine-derived fungus Penicillium concentricum ZLQ-69. J. Antibiot. 2015, 68, 748-751. [CrossRef] [PubMed]

173. Yamazaki, H.; Nakayama, W.; Takahashi, O.; Kirikoshi, R.; Izumikawa, Y.; Iwasaki, K.; Toraiwa, K.; Ukai, K.; Rotinsulu, H.; Wewengkang, D.S.; et al. Verruculides A and B, two new protein tyrosine phosphatase 1B inhibitors from an Indonesian ascidian-derived Penicillium verruculosum. Bioorg. Med. Chem. Lett. 2015, 25, 3087-3090. [CrossRef] [PubMed]

174. Lynn, D.G.; Phillips, N.J.; Hutton, W.C.; Shabanowitz, J.; Fennell, D.I.; Cole, R.J. Talaromycins: Application of homonuclear spin correlation maps to structure assignment. J. Am. Chem. Soc. 1982, 104, 7319-7322. [CrossRef]

175. Frisvad, J.C.; Filtenborg, O.; Samson, R.A.; Stolk, A.C. Chemotaxonomy of the genus Talaromyces. Antonie Van Leeuwenhoek 1990, 57, 179-189. [CrossRef] [PubMed]

176. Gao, Z.; Li, B.; Zheng, C.; Wang, G. Molecular detection of fungal communities in the Hawaiian marine sponges Suberites zeteki and Mycale armata. Appl. Environ. Microbiol. 2008, 74, 6091-6101. [CrossRef] [PubMed]

177. Webster, N.S.; Taylor, M.W. Marine sponges and their microbial symbionts: Love and other relationships. Environ. Microbiol. 2012, 14, 335-346. [CrossRef] [PubMed]

178. Gosio, B. Ricerche bacteriologiche e chimiche sulle alterazioni del mais. Riv. Ig. Sanità Pubblica 1896, 7, 825-868. 
179. Clutterbuck, P.W.; Raistrick, H. Studies in the biochemistry of microorganisms XXXI. The molecular constitution of the metabolic products of Penicillium brevi-compactum Dierckx and related species. Biochem. J. 1933, 27, 654-667. [CrossRef] [PubMed]

180. Lipsky, J.L. Mycophenolate mofetil. Lancet 1996, 348, 1357-1359. [CrossRef]

181. Bentley, R. Mycophenolic acid: A one hundred year odyssey from antibiotic to immunosuppressant. Chem. Rev. 2000, 100, 3801-3826. [CrossRef] [PubMed]

182. Oxford, A.E.; Raistrick, H.; Simonart, P. Studies in the biochemistry of microorganisms. LX. Griseofulvin, C17H17O6Cl, a metabolic product of Penicillium griseo-fulvum Dierckx. Biochem. J. 1939, 33, 240-248. [CrossRef] [PubMed]

183. Nicoletti, R.; Fiorentino, A. Antitumor metabolites of fungi. Curr. Bioact. Compd. 2014, 10, 207-244. [CrossRef]

184. Johnson, J.R.; Bruce, W.F.; Dutcher, J.D. Gliotoxin, the antibiotic principle of Gliocladium fimbriatum. I. Production, physical and biological properties. J. Am. Chem. Soc. 1943, 65, 2005-2009. [CrossRef]

185. Sekita, S.; Yoshihira, K.; Natori, S.; Kuwano, H. Structures of chaetoglobosin A and B, cytotoxic metabolites of Chaetomium globosum. Tetrahedron Lett. 1973, 14, 2109-2112. [CrossRef]

186. De Stefano, S.; Nicoletti, R.; Milone, A.; Zambardino, S. 3-O-Methylfunicone, a fungitoxic metabolite produced by the fungus Penicillium pinophilum. Phytochemistry 1999, 52, 1399-1401. [CrossRef]

187. Nicoletti, R.; de Stefano, M.; de Stefano, S.; Trincone, A.; Marziano, F. Antagonism against Rhizoctonia solani and fungitoxic metabolite production by some Penicillium isolates. Mycopathologia 2004, 158, 465-474. [CrossRef] [PubMed]

188. Stammati, A.; Nicoletti, R.; De Stefano, S.; Zampaglioni, F.; Zucco, F. Cytostatic properties of a novel compound derived from Penicillium pinophilum: An in vitro study. Altern. Lab. Anim. 2002, 30, 1-7.

189. Buommino, E.; Nicoletti, R.; Gaeta, G.M.; Orlando, M.; Ciavatta, M.L.; Baroni, A.; Tufano, M.A. 3-O-Methylfunicone induces apoptosis and hsp70 activation in HeLa cells. Cell Prolif. 2004, 37, 413-426. [CrossRef] [PubMed]

190. Buommino, E.; Boccellino, M.; De Filippis, A.; Petrazzuolo, M.; Cozza, V.; Nicoletti, R.; Ciavatta, M.L.; Quagliuolo, L.; Tufano, M.A. 3-O-methylfunicone produced by Penicillium pinophilum affects cell motility of breast cancer cells, downregulating $\alpha v \beta 5$ integrin and inhibiting metalloproteinase-9 secretion. Mol. Carcinog. 2007, 46, 930-940. [CrossRef]

191. Nicoletti, R.; Buommino, E.; De Filippis, A.; Lopez-Gresa, M.P.; Manzo, E.; Carella, A.; Petrazzuolo, M.; Tufano, M.A. Bioprospecting for antagonistic Penicillium strains as a resource of new antitumor compounds. World J. Microbiol. Biotechnol. 2008, 24, 189-195. [CrossRef]

192. Baroni, A.; De Luca, A.; De Filippis, A.; Petrazzuolo, M.; Manente, L.; Nicoletti, R.; Tufano, M.A.; Buommino, E. 3-O-Methylfunicone, a metabolite from Penicillium pinophilum, inhibits proliferation of human melanoma cells by causing G2/M arrest and inducing apoptosis. Cell Prolif. 2009, 42, 541-553. [CrossRef] [PubMed]

193. Nicoletti, R.; Manzo, E.; Ciavatta, M.L. Occurence and bioactivities of funicone-related compounds. Int. J. Mol. Sci. 2009, 10, 1430-1444. [CrossRef] [PubMed]

194. Buommino, E.; Paoletti, I.; De Filippis, A.; Nicoletti, R.; Ciavatta, M.L.; Menegozzo, S.; Menegozzo, M.; Tufano, M.A. 3-O-Methylfunicone, a metabolite produced by Penicillium pinophilum, modulates ERK1/2 activity, affecting cell motility of human mesothelioma cells. Cell Prolif. 2010, 43, 114-123. [CrossRef] [PubMed]

195. Buommino, E.; Tirino, V.; De Filippis, A.; Silvestri, F.; Nicoletti, R.; Ciavatta, M.L.; Pirozzi, G.; Tufano, M.A. 3-O-methylfunicone, from Penicillium pinophilum, is a selective inhibitor of breast cancer stem cells. Cell Prolif. 2011, 44, 401-409. [CrossRef] [PubMed]

196. Buommino, E.; De Filippis, A.; Nicoletti, R.; Menegozzo, M.; Menegozzo, S.; Ciavatta, M.L.; Rizzo, A.; Brancato, V.; Tufano, M.A.; Donnarumma, G. Cell-growth and migration inhibition of human mesothelioma cells induced by 3-O-methylfunicone from Penicillium pinophilum and cisplatin. Investig. New Drugs 2012, 30, 1343-1351. [CrossRef] [PubMed]

197. Nicoletti, R.; Scognamiglio, M.; Fiorentino, A. Structural and bioactive properties of 3-O-methylfunicone. Mini Rev. Med. Chem. 2014, 14, 1043-1047. [CrossRef]

198. Harned, A.M.; Volp, K.A. The sorbicillinoid family of natural products: Isolation, biosynthesis, and synthetic studies. Nat. Prod. Rep. 2011, 28, 1790-1810. [CrossRef] [PubMed]

199. Cram, D.J. Mold metabolites. II. The structure of sorbicillin, a pigment produced by the mold Penicillium notatum. J. Am. Chem. Soc. 1948, 70, 4240-4243. [CrossRef] [PubMed] 
200. Visagie, C.M.; Houbraken, J.; Frisvad, J.C.; Hong, S.B.; Klaassen, C.H.W.; Perrone, G.; Seifert, K.A.; Varga, J.; Yaguchi, T.; Samson, R.A. Identification and nomenclature of the genus Penicillium. Stud. Mycol. 2014, 78, 343-371. [CrossRef] [PubMed]

201. Matallah-Boutiba, A.; Ruiz, N.; Sallenave-Namont, C.; Grovel, O.; Amiard, J.C.; Pouchus, Y.F.; Boutiba, Z. Screening for toxigenic marine-derived fungi in Algerian mussels and their immediate environment. Aquaculture 2012, 342, 75-79. [CrossRef]

202. Greve, H.; Mohamed, I.E.; Pontius, A.; Kehraus, S.; Gross, H.; König, G.M. Fungal metabolites: Structural diversity as incentive for anticancer drug development. Phytochem. Rev. 2010, 9, 537-545. [CrossRef]

203. Gomes, N.G.; Lefranc, F.; Kijjoa, A.; Kiss, R. Can some marine-derived fungal metabolites become actual anticancer agents? Mar. Drugs 2015, 13, 3950-3991. [CrossRef] [PubMed]

204. Kakeya, H.; Onozawa, C.; Sato, M.; Arai, K.; Osada, H. Neuritogenic effect of epolactaene derivatives on human neuroblastoma cells which lack high-affinity nerve growth factor receptors. J. Med. Chem. 1997, 40, 391-394. [CrossRef] [PubMed]

205. Perpelescu, M.; Kobayashi, J.I.; Furuta, M.; Ito, Y.; Izuta, S.; Takemura, M.; Suzuki, M.; Yoshida, S. Novel phenalenone derivatives from a marine-derived fungus exhibit distinct inhibition spectra against eukaryotic DNA polymerases. Biochemistry 2002, 41, 7610-7616. [CrossRef] [PubMed]

206. Mizushina, Y.; Kobayashi, S.; Kuramochi, K.; Nagata, S.; Sugawara, F.; Sakaguchi, K. Epolactaene, a novel neuritogenic compound in human neuroblastoma cells, selectively inhibits the activities of mammalian DNA polymerases and human DNA topoisomerase II. Biochem. Biophys. Res. Commun. 2000, 273, 784-788. [CrossRef] [PubMed]

207. Nitiss, J.L. Targeting DNA topoisomerase II in cancer chemotherapy. Nat. Rev. Cancer 2009, 9, 338-350. [CrossRef] [PubMed]

208. Yamaguchi, K.; Tsuji, T.; Wakuri, S.; Yazawa, K.; Kondo, K.; Shigemori, H.; Kobayashi, J.I. Stimulation of nerve growth factor synthesis and secretion by fellutamide A in vitro. Biosci. Biotechnol. Biochem. 1993, 57, 195-199. [CrossRef] [PubMed]

209. Hines, J.; Groll, M.; Fahnestock, M.; Crews, C.M. Proteasome inhibition by fellutamide B induces nerve growth factor synthesis. Chem. Biol. 2008, 15, 501-512. [CrossRef] [PubMed]

210. Yang, X.; Du, L.; Tang, X.; Jung, S.Y.; Zheng, B.; Soh, B.Y.; Kim, S.Y.; Gu, Q.; Park, H. Brevicompanine E reduces lipopolysaccharide-induced production of proinflammatory cytokines and enzymes in microglia by inhibiting activation of activator protein-1 and nuclear factor-кB. J. Neuroimmunol. 2009, 216, 32-38. [CrossRef] [PubMed]

211. Chen, Y.P.; Yang, C.G.; Wei, P.Y.; Li, L.; Luo, D.Q.; Zheng, Z.H.; Lu, X.H. Penostatin derivatives, a novel kind of protein phosphatase $1 \mathrm{~B}$ inhibitors isolated from solid cultures of the entomogenous fungus Isaria tenuipes. Molecules 2014, 19, 1663-1671. [CrossRef] [PubMed]

212. Murray, A.P.; Faraoni, M.B.; Castro, M.J.; Alza, N.P.; Cavallaro, V. Natural AChE inhibitors from plants and their contribution to Alzheimer's disease therapy. Curr. Neuropharmacol. 2013, 11, 388-413. [CrossRef] [PubMed]

213. Lin, G.; Li, D.; Chidawanyika, T.; Nathan, C.; Li, H. Fellutamide B is a potent inhibitor of the Mycobacterium tuberculosis proteasome. Arch. Biochem. Biophys. 2010, 501, 214-220. [CrossRef] [PubMed]

214. Zhao, J.C.; Li, X.M.; Gloer, J.B.; Wang, B.G. First total syntheses and antimicrobial evaluation of penicimonoterpene, a marine-derived monoterpenoid, and its various derivatives. Mar. Drugs 2014, 12, 3352-3370. [CrossRef] [PubMed]

215. Hayashi, H.; Matsumoto, H.; Akiyama, K. New insecticidal compounds, communesins C, D and E, from Penicillium expansum Link MK-57. Biosci. Biotechnol. Biochem. 2004, 68, 753-756. [CrossRef] [PubMed]

216. Park, M.S.; Fong, J.J.; Oh, S.Y.; Kwon, K.K.; Sohn, J.H.; Lim, Y.W. Marine-derived Penicillium in Korea: Diversity, enzyme activity, and antifungal properties. Antonie Van Leeuwenhoek 2014, 106, 331-345. [CrossRef] [PubMed]

217. Bringmann, G.; Gulder, T.A.; Lang, G.; Schmitt, S.; Stöhr, R.; Wiese, J.; Nagel, K.; Imhoff, J.F. Large-scale biotechnological production of the antileukemic marine natural product sorbicillactone A. Mar. Drugs 2007, 5, 23-30. [CrossRef] [PubMed]

(C) 2016 by the authors; licensee MDPI, Basel, Switzerland. This article is an open access article distributed under the terms and conditions of the Creative Commons by Attribution (CC-BY) license (http://creativecommons.org/licenses/by/4.0/). 